\title{
Thermal Effects of Asymmetric Electrolytes in Electric Double Layer Capacitors
}

\author{
Anna L. d'Entremont and Laurent Pilon* \\ University of California, Los Angeles, \\ Henry Samueli School of Engineering and Applied Science, \\ Mechanical and Aerospace Engineering Department, \\ 420 Westwood Plaza, \\ Los Angeles, CA 90095, USA
}

\begin{abstract}
This study presents a thermal model, derived from first principles, for electric double layer capacitors (EDLCs) with multiple ion species and/or asymmetric electrolytes. It accounts for both irreversible and reversible heat generation rates resulting from the transient electrodiffusion of ions within the electrolyte. Detailed numerical simulations of EDLCs with planar electrodes and binary and asymmetric electrolytes were performed under galvanostatic cycling. The irreversible Joule heating decreased with increasing valency and/or diffusion coefficient of either ion. The local reversible heat generation rate near a given electrode was determined by the properties of the counterion. It increased with increasing counterion valency and/or decreasing counterion diameter. As a result, the electrode with the counterion of smaller diameter and/or larger valency experienced significantly larger temperature oscillations during galvanostatic cycling than the opposite electrode. In general, EDLC electrolytes featuring ions with large valency and/or small diameter produce large capacitance but also large reversible heating. The present study suggests that EDLC electrolytes should feature large bulk ion concentrations and at least one ion with a large diffusion coefficient to minimize both irreversible and reversible heating.
\end{abstract}

Keywords: supercapacitors, energy storage, Joule heating, reversible heating

*Corresponding author, Email: pilon@seas.ucla.edu, Tel: +1 (310) 206-5598, Fax: +1 (310) 2062302 


\section{Introduction}

Electric double layer capacitors (EDLCs) are promising electrical energy storage devices for applications requiring large power density, rapid response, or long cycle life [1-3]. Such applications include regenerative braking, load leveling, and dynamic stabilization of the utility grid $[2,4-7]$. EDLCs physically store electric charge within the electric double layer (EDL) forming at the electrode/electrolyte interface. Their electrical performance lies between that of batteries and of dielectric capacitors [1-3]. EDLC power densities, cycle life, and cycle efficiencies are typically much larger than those of batteries $[1,3]$. In addition, their porous electrodes with small charge separation distances result in much larger energy densities than those of dielectric capacitors [1-3].

EDLCs exhibit both irreversible and reversible heat generation during operation $[5,8]$. Excessive temperature rise in EDLCs causes various detrimental effects including accelerated ageing $[4-7,9,10]$ and increased self-discharge rates [5-7,9]. Thermal modeling can be used to predict operating temperature and to develop strategies to mitigate these effects.

The heat generation rate within the electrolyte of an EDLC is closely coupled with ion transport and electrolyte properties [8]. Previous thermal models of EDLCs accounting for reversible heating $[5,8,10-12]$ were limited to binary and symmetric electrolytes, i.e., the electrolytes had only two ion species with identical valency, effective ion diameter, and diffusion coefficient $[5,8,13]$. However, many widely used electrolytes are asymmetric, such as aqueous $\mathrm{H}_{2} \mathrm{SO}_{4}$ [13-16]. In addition, electrolyte mixtures including more than two ion species have attracted interest for EDLC applications because certain mixtures perform better than either of the original electrolytes. For example, eutectic mixtures of ionic liquids can provide broader operating temperature ranges than either constituent [17-19]. These asymmetric and/or multi-species electrolytes cannot be rigorously accounted for by existing thermal models.

The present study aims to develop a general thermal model of EDLCs from first principles accounting for multi-species and/or asymmetric electrolytes. It extends our previous thermal analysis of EDLCs with binary and symmetric electrolytes [8]. In addition, detailed numerical 
simulations of binary and asymmetric electrolytes were performed to investigate effects of electrolyte asymmetry on the irreversible and reversible heat generation rates within the EDLCs and the resulting local temperature.

\section{Background}

\subsection{Electric Double Layer Structure}

Figure 1(a) illustrates the electric double layer (EDL) structure of a binary and asymmetric electrolyte according to the Stern model [20, 21]. The compact Stern layer adjacent to the electrode surface contains no free charge because its boundary at $x=H$ marks the distance of closest approach for a solvated ion to the electrode surface [20-22]. Within the diffuse layer, ions are mobile under the competing influences of electrostatic forces, diffusion, and steric effects $[20,21,23]$. The EDL is the region near the electrode with a net space charge density in contrast to the electrically neutral bulk electrolyte. Its thickness varies with electrolyte concentration, ion valencies, and temperature [20,21]. The net ionic charge

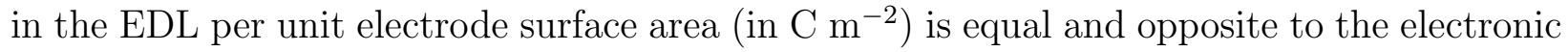
surface charge density in the electrode so that the region around the interface is electrically neutral overall [20-22].

\subsection{Capacitance}

Capacitance characterizes the amount of electric charge stored in an EDLC as a function of its cell potential. For galvanostatic cycling consisting of alternating charging and discharging steps at a constant current density $\pm j_{s}=d q_{s} / d t$, the differential and integral capacitances can be expressed as $[1,24]$

$$
C_{s, d i f f}=\frac{d q_{s}}{d \psi_{s}}=\frac{j_{s}}{d \psi_{s} / d t} \quad \text { and } \quad C_{s, i n t}=\frac{q_{s}}{\psi_{s}}=\frac{j_{s} t_{c} / 2}{\psi_{\max }-\psi_{\min }}
$$

where $q_{s}$ is the surface charge density (in $\mathrm{C} \mathrm{m}^{-2}$ ), $t_{c}$ is the cycle period, and $\psi_{s}$ is the cell potential (in V) with upper and lower limits $\psi_{\max }$ and $\psi_{\min }$. 


\subsection{Modeling of Ion Transport}

Previous studies have demonstrated the importance of accounting for the finite size of ions for accurate modeling of electrodiffusion at large electrolyte concentrations and/or large voltages [25]. However, many of the existing ion transport models accounting for finite ion size are limited to binary and/or symmetric electrolytes [23, 26-33]. The generalized modified Poisson-Nernst-Planck (GMPNP) model developed by Wang et al. [13]predicts the local electric potential and ion concentrations in asymmetric and multi-species electrolytes with finite ion size. The electric potential $\psi(\mathbf{r}, t)$ satisfies the Poisson equation, expressed as [13]

$$
-\nabla \cdot\left(\epsilon_{0} \epsilon_{r} \nabla \psi\right)= \begin{cases}0 & \text { in the Stern layers } \\ F \sum_{i=1}^{n} z_{i} c_{i} & \text { in the diffuse layer }\end{cases}
$$

where $\epsilon_{0}=8.854 \times 10^{-12} \mathrm{~F} \mathrm{~m}^{-1}$ and $\epsilon_{r}$ are the vacuum permittivity and the relative permittivity of the electrolyte, respectively. The Faraday constant is denoted by $F$ and equal to $96485 \mathrm{C} \mathrm{mol}^{-1}$. The valency and concentration of ion species $i$ are denoted by $z_{i}$ and $c_{i}$, respectively, while $n$ is the number of ion species in the solution. Each ion concentration $c_{i}(\mathbf{r}, t)$ in the diffuse layer is governed by the mass conservation equation expressed as [13]

$$
\frac{\partial c_{i}}{\partial t}=-\nabla \cdot \mathbf{N}_{i} \quad \text { with } \quad \mathbf{N}_{i}=-D_{i} c_{i}\left[\frac{z_{i} F}{R_{u} T} \nabla \psi+\nabla \ln \left(\gamma_{i, L} c_{i}\right)\right]
$$

where $\mathbf{N}_{i}$ is the flux of ion species $i$ in mol m $\mathrm{m}^{-2} \mathrm{~s}^{-1}, D_{i}$ is the diffusion coefficient of ion species $i, R_{u}=8.314 \mathrm{~J} \mathrm{~K}^{-1} \mathrm{~mol}^{-1}$ is the universal gas constant, and $T$ is the absolute temperature. The activity coefficient $\gamma_{i, L}$ was assumed to obey a Langmuir-type law accounting for the excluded volume due to finite ion size and expressed as $[13,34]$

$$
\gamma_{i, L}=\frac{1}{1-\sum_{i=1}^{n} \frac{c_{i}}{c_{i, \max }}}
$$

Here, $c_{i, \max }=1 / N_{A} a_{i}^{3}$ is the theoretical maximum concentration of ion species $i$ assuming simple cubic packing of ions with effective ion diameter $a_{i}$ while $N_{A}=6.022 \times 10^{23} \mathrm{~mol}^{-1}$ is the Avogadro constant. The ion flux can then be expressed as [13]

$$
\mathbf{N}_{i}=-\frac{D_{i} F z_{i} c_{i}}{R_{u} T} \nabla \psi-D_{i} \nabla c_{i}-\frac{D_{i} N_{A} c_{i}}{1-N_{A} \sum_{i=1}^{n} a_{i}^{3} c_{i}} \sum_{i=1}^{n} a_{i}^{3} \nabla c_{i} .
$$


Here, the derivation assumed that the effective ion diameters $a_{i}$ are independent of location, i.e., they do not vary with the local ion concentrations or temperature. The first, second, and third terms on the right-hand side of Equation (5) correspond to the ion fluxes due to electromigration, diffusion, and steric effects, respectively.

\subsection{Thermal Modeling of EDLCs with Binary and Symmetric Electrolytes}

In our previous study [8], we used the MPNP model to derive a first-principles thermal model of EDLCs with binary and symmetric electrolytes. The electrolyte was assumed to have negligible bulk motion, no chemical reactions, and negligible energy flux due to the Dufour effect, i.e., driven by gradients of electrochemical potential and/or pressure [22, 35, 36]. Then, the energy conservation equation for a control volume of electrolyte in terms of the temperature $T$ was derived as [8]

$$
\rho c_{p} \frac{\partial T}{\partial t}=\nabla \cdot(k \nabla T)+\dot{q}
$$

where $\rho, c_{p}$, and $k$ are the density, specific heat, and thermal conductivity of the electrolyte,

respectively. Here, $\dot{q}(\mathbf{r}, t)$ is the local volumetric heat generation rate (in $\mathrm{W} \mathrm{m}^{-3}$ ) consisting of the sum of the heat generation rates (i) $\dot{q}_{E}$ arising from ions decreasing their electrical potential energy and (ii) $\dot{q}_{S}$ caused by ion fluxes along gradients of chemical potential, partial molar entropy, and temperature also called the "heat of mixing" [8, 37]. For binary and symmetric electrolytes, $\dot{q}_{E}$ consists of three contributions corresponding to Joule heating and to heating arising from ion diffusion and steric effects [8]. The heat of mixing $\dot{q}_{S}$ has two contributions arising from concentration gradients and from temperature gradients [8]. Aside from the Joule heating, which is always positive and therefore irreversible, the other contributions to the heat generation rate can be positive or negative and constitute the overall reversible heat generation rate $\dot{q}_{r e v}$ such that $\dot{q}=\dot{q}_{i r r}+\dot{q}_{r e v}$. The expressions for $\dot{q}_{i r r}$ and $\dot{q}_{\text {rev }}$ for binary and symmetric electrolytes can be found in Ref. [8].

Note that Biesheuvel et al. [38] discussed a heat generation rate corresponding to $\dot{q}_{E}$ within the electrolyte of ion exchange membranes. The authors observed that it could be either positive or negative within the EDLs, depending on the direction of the current relative 
to the local electric field, and described the cooling phenomenon as "negative Joule heating" [38]. By contrast, the present study reserves the term "Joule heating" for the irreversible contribution $\dot{q}_{i r r}$ by analogy to the Joule heating in ohmic conductors originally defined by Joule [39].

\section{Analysis}

\subsection{Schematic and Assumptions}

Figure 1(b) illustrates the one-dimensional EDLC simulated in the present study. It consists of two planar electrodes separated by an electrolyte with inter-electrode spacing 2L. The electrodes located at $x=0$ and $x=2 L$ are denoted as electrodes $\mathrm{A}$ and $\mathrm{B}$, respectively. The ion species with the largest effective ion diameter corresponds to $i=1$. In contrast to EDLC cells using symmetric electrolytes, those using asymmetric electrolytes lack antisymmetry in the electric potential and ion concentrations [13]. Thus, for asymmetric electrolytes, the entire electrolyte region must be simulated [13].

To make the problem mathematically tractable, the following assumptions were made: (1) bulk motion of the electrolyte was negligible, (2) the effective ion diameters were independent of local ion concentrations and temperature, as previously mentioned, (3) non-electrostatic ion adsorption was negligible, (4) the Stern layer thickness $H$ was equal to the effective radius of the largest ion species, i.e., $H=a_{1} / 2$, and (5) the EDLC was thermally insulated.

\subsection{Heat Generation in Multi-Species and Asymmetric Electrolytes}

The electrical heat generation rate $\dot{q}_{E}$ is defined as [8]

$$
\dot{q}_{E}=\mathbf{j} \cdot \mathbf{E}
$$

where $\mathbf{j}=\sum_{i=1}^{n} z_{i} F \mathbf{N}_{i}$ is the ionic current density [22] and $\mathbf{E}=-\nabla \psi$ is the electric field vector. Based on the expression for the ion flux $\mathbf{N}_{i}$ in the GMPNP model [Equation (5)], the current density $\mathbf{j}$ in an asymmetric electrolyte solution can be written as

$$
\mathbf{j}=\sigma \mathbf{E}-F \sum_{i=1}^{n} D_{i} z_{i} \nabla c_{i}-\left(\frac{F N_{A} \sum_{i=1}^{n} D_{i} z_{i} c_{i}}{1-N_{A} \sum_{i=1}^{n} a_{i}^{3} c_{i}}\right) \sum_{i=1}^{n} a_{i}^{3} \nabla c_{i}
$$


Here, $\sigma$ is the electrical conductivity of the electrolyte expressed as [22, 40]

$$
\sigma=\frac{F^{2}}{R_{u} T} \sum_{i=1}^{n} D_{i} z_{i}^{2} c_{i} .
$$

Equation (8) can be rearranged to find an expression for the electric field vector $\mathbf{E}$ to be substituted into Equation (7). This results in three contributions to $\dot{q}_{E}$ such that $\dot{q}_{E}=$ $\dot{q}_{i r r}+\dot{q}_{E, d}+\dot{q}_{E, s}$. The first term $\dot{q}_{i r r}$ corresponds to the irreversible Joule heating and is expressed as

$$
\dot{q}_{i r r}=\frac{|\mathbf{j}|^{2}}{\sigma} .
$$

The heat generation rates $\dot{q}_{E, d}$ and $\dot{q}_{E, s}$ are reversible and arise from ion diffusion and steric repulsion, respectively. For asymmetric electrolytes with $n$ ion species, they are expressed as

$$
\dot{q}_{E, d}=\frac{\mathbf{j}}{\sigma} \cdot\left(F \sum_{i=1}^{n} D_{i} z_{i} \nabla c_{i}\right) \quad \text { and } \quad \dot{q}_{E, s}=\frac{\mathbf{j}}{\sigma} \cdot \frac{\left(F \sum_{i=1}^{n} D_{i} z_{i} c_{i}\right)\left(N_{A} \sum_{i=1}^{n} a_{i}^{3} \nabla c_{i}\right)}{\left(1-N_{A} \sum_{i=1}^{n} a_{i}^{3} c_{i}\right)} .
$$

Note that $\dot{q}_{E, d}$ and $\dot{q}_{E, s}$ differ from zero only in the presence of an ion concentration gradient $\nabla c_{i}$.

The heat of mixing $\dot{q}_{S}$ is defined as [8]

$$
\dot{q}_{S}=\sum_{i=0}^{n} \mathbf{N}_{i} \cdot R_{u} \nabla\left(T^{2} \frac{\partial \ln \gamma_{i, D H}}{\partial T}\right)
$$

where $\gamma_{i, D H}$ is the activity coefficient of ion species $i$ or of the solvent $i=0$. The present study uses the expression of $\gamma_{i, D H}$ given by the Debye-Hückel limiting law as it accounts for the effects of both temperature and ion concentrations. It was derived for dilute electrolytes and accounts for long-range electrical interactions between ions and for thermal agitation while neglecting short-range ion-solvent or ion-ion interactions [22, 41]. Then, the activity coefficient of ion species $i$ can be expressed as [22, 41]

$$
\ln \gamma_{i, D H}=-\frac{z_{i}^{2} e F^{2}\left(\sum_{i=1}^{n} z_{i}^{2} c_{i}\right)^{1 / 2}}{8 \pi\left(\epsilon_{0} \epsilon_{r} R_{u} T\right)^{3 / 2}} .
$$


Substituting Equation (13) into the expression for the heat of mixing $\dot{q}_{S}$ given by Equation (12) and assuming negligible contribution from the solvent $(i=0)$ yields two terms such that $\dot{q}_{S}=\dot{q}_{S, c}+\dot{q}_{S, T}$, where $\dot{q}_{S, c}$ and $\dot{q}_{S, T}$ are expressed as

$$
\begin{gathered}
\dot{q}_{S, c}=\frac{3}{32 \pi} \frac{e F^{2}}{\left(\epsilon_{0} \epsilon_{r}\right)^{3 / 2}\left(R_{u} T \sum_{i=1}^{n} z_{i}^{2} c_{i}\right)^{1 / 2}}\left(\sum_{i=1}^{n} z_{i}^{2} \mathbf{N}_{i}\right) \cdot\left(\sum_{i=1}^{n} z_{i}^{2} \nabla c_{i}\right) \\
\text { and } \dot{q}_{S, T}=-\frac{3}{32 \pi} \frac{e F^{2}\left(\sum_{i=1}^{n} z_{i}^{2} c_{i}\right)^{1 / 2}}{\left(\epsilon_{0} \epsilon_{r}\right)^{3 / 2} R_{u}^{1 / 2} T^{3 / 2}} \sum_{i=1}^{n} z_{i}^{2} \mathbf{N}_{i} \cdot \nabla T .
\end{gathered}
$$

These terms correspond to the heat of mixing arising from concentration gradients and from temperature gradients, respectively. Here, $\dot{q}_{S, c}$ represents heat released by ions migrating in the direction of increasing concentration, e.g., into the EDL region, as this leads to a more ordered distribution of ions and consequently smaller entropy. Meanwhile, $\dot{q}_{S, T}$ represents heat released by ions migrating in the direction of decreasing temperature, as smaller thermal agitation results in smaller entropy. For binary and symmetric electrolytes, Equations (10), (11), and (14) simplify to those derived in Ref. [8].

Note that the expression for the activity coefficient $\gamma_{i, D H}$ differs from the Langmuirtype activity coefficient $\gamma_{i, L}$ used to derive the GMPNP model and given by Equation (4). The different formulations were used because the heat of mixing $\dot{q}_{S}$ must account for the temperature dependence of $\gamma_{i, D H}$ while the GMPNP derivation must account for the steric effects through $\gamma_{i, L}$. Unfortunately, neither $\gamma_{i, L}$ nor $\gamma_{i, D H}$ captures all these effects simultaneously. However, the steric repulsion represented by the Langmuir-type activity coefficient $\gamma_{i, L}$ does affect the heat generation rates through its influence on the ion concentrations $c_{i}$, the ion fluxes $\mathbf{N}_{i}$, and the current density $\mathbf{j}$ appearing in the expressions of $\dot{q}_{i r r}, \dot{q}_{E, d}, \dot{q}_{E, s}, \dot{q}_{S, c}$, and $\dot{q}_{S, T}$

Overall, the total heat generation rate $\dot{q}$ can be expressed as the sum of an irreversible $\dot{q}_{i r r}$ and a reversible $\dot{q}_{r e v}$ heat generation rate such that $\dot{q}=\dot{q}_{i r r}+\dot{q}_{r e v}$ where $\dot{q}_{r e v}=\dot{q}_{E, d}+$ $\dot{q}_{E, s}+\dot{q}_{S, c}+\dot{q}_{S, T}$. 


\subsection{Initial and Boundary Conditions}

The one-dimensional governing Equation (2) for $\psi(x, t)$ was solved within the two Stern layers and the diffuse layer. It required one initial condition and two boundary conditions for each region. Initially, the potential was uniform and equal to zero such that

$$
\psi(x, 0)=0 \mathrm{~V} \text { for } 0 \leq x \leq 2 L
$$

During galvanostatic cycling, the electric current density at electrode A alternated between charging at current density $+j_{s}$ and discharging at current density $-j_{s}$ as a square wave of cycle period $t_{c}$. Charge conservation requires the displacement current density $j_{d}$ in the electrolyte at the surface of electrode A to be equal to the electronic current density within the electrode. This condition was expressed as

$$
j_{d}=-\epsilon_{0} \epsilon_{r} \frac{\partial^{2} \psi}{\partial t \partial x}(0, t)= \begin{cases}j_{s} & \text { for }\left(n_{c}-1\right) t_{c} \leq t<\left(n_{c}-1 / 2\right) t_{c} \\ -j_{s} & \text { for }\left(n_{c}-1 / 2\right) t_{c} \leq t<n_{c} t_{c}\end{cases}
$$

where $n_{c}=1,2, \ldots$ is the cycle number. Moreover, the electric potential and the electric field were continuous across the Stern/diffuse layer interface located at $x=H$ such that

$$
\psi\left(H^{-}, t\right)=\psi\left(H^{+}, t\right) \quad \text { and } \quad \frac{\partial \psi}{\partial x}\left(H^{-}, t\right)=\frac{\partial \psi}{\partial x}\left(H^{+}, t\right)
$$

Similarly, they were continuous across the Stern/diffuse layer interface at $x=2 L-H$ so that

$$
\psi\left(2 L-H^{-}, t\right)=\psi\left(2 L-H^{+}, t\right) \quad \text { and } \quad \frac{\partial \psi}{\partial x}\left(2 L-H^{-}, t\right)=\frac{\partial \psi}{\partial x}\left(2 L-H^{+}, t\right)
$$

Finally, the surface of electrode B was electrically grounded, i.e., $\psi(2 L, t)=0 \mathrm{~V}$.

The one-dimensional mass conservation Equation (3) for ion concentration $c_{i}(x, t)$ was solved only within the diffuse layer since the Stern layer does not contain free charge [20, 21]. Thus, one initial condition and two boundary conditions were required. The ion concentrations were initially uniform, and the solution was electrically neutral, i.e.,

$$
c_{i}(x, 0)=c_{i, \infty} \quad \text { with } \quad \sum_{i=1}^{n} z_{i} c_{i, \infty}=0 .
$$


At the Stern/diffuse layer interfaces located at $x=H$ and $x=2 L-H$, all ion fluxes vanished because there was no ion insertion into the electrodes, i.e.,

$$
N_{i}(H, t)=N_{i}(2 L-H, t)=0 \mathrm{~mol} \mathrm{~m}{ }^{-2} \mathrm{~s}^{-1}
$$

The one-dimensional energy conservation Equation (6) expressed in terms of the temperature $T(x, t)$ was solved within the two Stern layers and the diffuse layer. It required one initial condition and two boundary conditions for each region. The initial temperature was uniform and equal to $T(x, 0)=T_{0}$. The surfaces of electrode $\mathrm{A}$ at $x=0$ and of electrode B at $x=2 L$ were assumed to be thermally insulated such that

$$
-k \frac{\partial T}{\partial x}(0, t)=0 \mathrm{~W} \mathrm{~m}^{-2} \text { and } \quad-k \frac{\partial T}{\partial x}(2 L, t)=0 \mathrm{~W} \mathrm{~m}^{-2}
$$

The temperature and heat flux were continuous across the Stern/diffuse layer interface at $x=H$, i.e.,

$$
T\left(H^{-}, t\right)=T\left(H^{+}, t\right) \quad \text { and } \quad-k \frac{\partial T}{\partial x}\left(H^{-}, t\right)=-k \frac{\partial T}{\partial x}\left(H^{+}, t\right) .
$$

Similarly, they were continuous across the second Stern/diffuse layer interface at $x=2 L-H$ so that

$$
T\left(2 L-H^{-}, t\right)=T\left(2 L-H^{+}, t\right) \quad \text { and } \quad-k \frac{\partial T}{\partial x}\left(2 L-H^{-}, t\right)=-k \frac{\partial T}{\partial x}\left(2 L-H^{+}, t\right) .
$$

\subsection{Constitutive Relationships}

The electrolytes simulated were based on the properties of aqueous $\mathrm{H}_{2} \mathrm{SO}_{4}$, a common binary and asymmetric electrolyte used in EDLCs [1]. The ion valencies for aqueous $\mathrm{H}_{2} \mathrm{SO}_{4}$ were equal to $z_{1}=-2$ and $z_{2}=1$, where $i=1$ and $i=2$ corresponded to $\mathrm{SO}_{4}^{2-}$ and $\mathrm{H}^{+}$, respectively. The associated solvated ion diameters were taken as $a_{1}=0.76 \mathrm{~nm}$ and $a_{2}=$ $0.56 \mathrm{~nm}$ and the diffusion coefficients as $D_{1}=1.1 \times 10^{-9} \mathrm{~m}^{2} \mathrm{~s}^{-1}$ and $D_{2}=9.3 \times 10^{-9} \mathrm{~m}^{2} \mathrm{~s}^{-1}$ [14-16]. It is evident that aqueous $\mathrm{H}_{2} \mathrm{SO}_{4}$ was significantly asymmetric with respect to valency, ion diameter, and diffusion coefficient. Finally, the relative permittivity $\epsilon_{r}=78.4$, density $\rho=997 \mathrm{~kg} \mathrm{~m}^{-3}$, specific heat $c_{p}=4180 \mathrm{~J} \mathrm{~kg}^{-1} \mathrm{~K}^{-1}$, and thermal conductivity $k=0.61 \mathrm{~W} \mathrm{~m}^{-1} \mathrm{~K}^{-1}$ were taken as those of water [14]. 


\subsection{Method of Solution}

The one-dimensional governing Equations (2), (3), and (6) and the associated initial and boundary conditions were solved numerically using finite element methods. The numerical convergence of the solution was assessed based on the predicted potential $\psi(x, t)$, ion concentrations $c_{1}(x, t)$ and $c_{2}(x, t)$, and temperature $T(x, t)$. The temperature was the most sensitive to the choice of mesh element size and time step. The mesh element size was the smallest at the Stern/diffuse interfaces due to the large gradients of potential and concentrations in this region and gradually increased away from these boundaries. The mesh was refined by reducing the element size at the Stern/diffuse layer interface and by reducing the maximum element growth rate. The time step was controlled by the relative and absolute time tolerances [42]. At each time step, the estimated local error between the solutions at the previous and the current time step was compared with the time tolerances. The time step was then adjusted until the convergence criterion was satisfied, as described in Ref. [42]. This enabled the use of small time steps during periods of rapid changes in $\psi(x, t), c_{i}(x, t)$, and/or $T(x, t)$, while using a larger time step for the rest of the simulation. The numerical solution was considered converged when halving (i) the element size at the Stern/diffuse layer interface, (ii) the maximum element growth rate, and (iii) both the relative and absolute tolerances resulted in less than $0.5 \%$ maximum relative difference in the local temperature rise $T(x, t)-T_{0}$.

\section{Results and Discussion}

Table 1 summarizes the cases simulated to investigate the effect of asymmetric valencies $z_{i}$, ion diameters $a_{i}$, and diffusion coefficients $D_{i}$ on the thermal behavior of EDLCs with binary electrolytes. Case 1 represents a symmetric electrolyte with ion properties corresponding to those of $\mathrm{H}^{+}$ions and was used as a baseline case. Cases 2-4 were chosen to illustrate the effect of valency $z_{i}$ with all other properties equal to those of Case 1 . Case 2 corresponded to symmetric electrolyte with $-z_{1}=z_{2}=2$ based on the valency of $\mathrm{SO}_{4}^{2-}$ while Case 3 and 4 represented asymmetric electrolytes with $z_{1}=-2$ and $z_{2}=1$. Asymmetry in 
valency required asymmetric bulk ion concentrations $c_{2, \infty}=-z_{1} c_{1, \infty} / z_{2}$ in order to satisfy electroneutrality. Thus, Cases 3 and 4 were chosen with different bulk concentrations $c_{i, \infty}$ so that $z_{1}$ and $c_{1, \infty}$ in Case 3 equaled those of Case 2, while $z_{2}$ and $c_{2, \infty}$ in Case 4 equaled those of Case 1.

Similarly, the effect of ion diameter $a_{i}$ was demonstrated by Case $5\left(a_{1}=a_{2}=0.76 \mathrm{~nm}\right)$ and Case $6\left(a_{1}=0.76 \mathrm{~nm}\right.$ and $\left.a_{2}=0.56 \mathrm{~nm}\right)$, while the effect of diffusion coefficient $D_{i}$ was shown in Case $7\left(D_{1}=D_{2}=1.1 \times 10^{-9} \mathrm{~m}^{2} \mathrm{~s}^{-1}\right)$ and Case $8\left(D_{1}=1.1 \times 10^{-9} \mathrm{~m}^{2} \mathrm{~s}^{-1}\right.$ and $\left.D_{2}=9.3 \times 10^{-9} \mathrm{~m}^{2} \mathrm{~s}^{-1}\right)$. The electrolyte relative permittivity $\epsilon_{r}$, density $\rho$, specific heat $c_{p}$, and thermal conductivity $k$ remained the same for all cases. The inter-electrode spacing and initial temperature were equal to $L=20 \mu \mathrm{m}$ and $T_{0}=298 \mathrm{~K}$, respectively. The cell was cycled galvanostatically at $j_{s}=14 \mathrm{~mA} \mathrm{~cm}^{-2}$ with cycle period $t_{c}=7.6 \mathrm{~ms}$. This current density $j_{s}$ was within the range of current densities per unit surface area of the current collector reported in experimental studies $[5,43]$. The combination of $j_{s}$ and $t_{c}$ was chosen to yield a maximum cell voltage of $1 \mathrm{~V}$ for the baseline Case 1. The current density and cycle period were held constant for all cases to facilitate comparison. The electrolyte properties were assumed to be constant and independent of temperature. The temperature in the expressions of $\mathbf{N}_{i}, \dot{q}_{S, c}$, and $\dot{q}_{S, T}$ was taken as the initial temperature $T_{0}$.

\subsection{Electric Potential}

Figure 2 shows the cell potential $\psi_{s}(t)=\psi(0, t)-\psi(2 L, t)$ computed as a function of time $t$ over one and a half cycles for (a) Cases 1-4 featuring different ion valencies $z_{i}$ and (b) Cases 1, 5, and 6 featuring different effective ion diameters. Results for Cases 7 and 8 featuring different diffusion coefficients $D_{i}$ were not shown because the predicted cell potentials overlapped that of Case $1\left(D_{1}=D_{2}=9.3 \times 10^{-9} \mathrm{~m}^{2} \mathrm{~s}^{-1}\right)$ at all times. In all cases, the cell potential increased during the charging step and decreased during the discharging step. As previously mentioned, the current density $j_{s}$ and cycle period $t_{c}$ were chosen so that the maximum cell potential for the baseline Case 1 would equal $1 \mathrm{~V}$. The numerically predicted cell potential qualitatively resembled those measured during galvanostatic cycling of EDLCs [43, 44]. Note that the measured cell potential often featured a significant "IR 
drop" due to ohmic resistance. However, the predicted cell potential, shown in Figure 2, was almost entirely due to the electric double layer formation. The portion of the cell potential due to ohmic resistance in the electrolyte can be approximated as $j_{s}(2 L) / \sigma_{\infty}$ where $\sigma_{\infty}$ is the electrical conductivity of the electrolyte given by Equation (9) with the bulk ion concentrations $c_{1, \infty}$ and $c_{2, \infty}$. This ohmic potential drop was on the order of $10^{-3}$ to $10^{-5} \mathrm{~V}$ and was less than $0.1 \%$ of the maximum cell potential for all Cases 1-8. The IR drop can be reproduced numerically by reducing the electrolyte conductivities $\sigma_{\infty}$, increasing the inter-electrode spacing, and/or accounting for the electrical resistance of the electrodes and current collectors (see supplementary material).

Figure 2(a) indicates that increasing either or both ion valencies $\left|z_{i}\right|$ resulted in a smaller cell potential at all times. For galvanostatic cycling, the surface charge density $q_{s}=j_{s} t_{c} / 2$ added during the charging step was the same for all cases. Thus, the integral capacitance $C_{s, i n t}$, given by Equation (1), increased with increasing $\left|z_{i}\right|$ (Table 1). In addition, Cases 3 and 4 featuring the same ion properties $z_{i}, a_{i}$, and $D_{i}$, but different bulk ion concentrations $c_{i, \infty}$ demonstrate that doubling $c_{i, \infty}$ slightly increased $C_{s, i n t}$. By contrast, Figure 2(b) indicates that increasing either or both effective ion diameters $a_{i}$ substantially increased the cell potential, corresponding to a decrease in capacitance $C_{s, \text { int }}$ (Table 1 ). These changes in integral capacitance with $\left|z_{i}\right|$ and $a_{i}$ were consistent with those observed from cyclic voltammetry simulations of EDLCs with binary and asymmetric electrolytes [13]. Table 1 also shows that decreasing either or both ion diffusion coefficients $D_{i}$ had no effect on $C_{s, i n t}$. This was also consistent with the results of cyclic voltammetry simulations outside the "diffusion-limited" regime [13].

\subsection{Concentrations}

Figure 3 shows (a) and (c) the anion concentration $c_{1}\left(x, 3 t_{c} / 2\right)$ near the positive electrode A as a function of location $x$ as well as (b) and (d) the cation concentration $c_{2}\left(x, 3 t_{c} / 2\right)$ near the negative electrode $\mathrm{B}$ as a function of $2 L-x$ at time $t=3 t_{c} / 2$ at the end of a charging step. Figures 3(a) and 3(b) show Cases 1-4 featuring different ion valencies $z_{i}$, while Figures 3(c) and 3(d) show Cases 1, 5, and 6 with different effective ion diameters $a_{i}$. 
Figure 3 indicates that electrolytes with asymmetric $z_{i}$ or $a_{i}$, e.g., those in Cases 3 , 4, and 6 , exhibited spatially asymmetric concentration profiles. Overall, the concentration profiles near each electrode were determined by the properties of the counterion, i.e., the anion (species 1) near the positive electrode [Figures 3(a) and 3(c)] and the cation (species 2) near the negative electrode [Figures 3(b) and 3(d)]. The ion diameter $a_{i}$ controlled the counterion concentration at the Stern/diffuse layer interfaces located at $x=H$ and $x=2 L-H$ where the counterion concentration reached its theoretical maximum $c_{i, \max }=1 / N_{A} a_{i}^{3}$. The maximum concentration gradient also decreased with increasing $a_{i}$ due to the smaller concentration drop between the Stern/diffuse layer interface and the bulk electrolyte. By contrast, increasing the counterion valency $\left|z_{i}\right|$ had no effect on the surface counterion concentration. However, it resulted in a thinner EDL and steeper concentration gradients near the electrodes. These effects can be attributed to the fact that (i) fewer ions were necessary to balance the same electrode charge and (ii) the electrostatic forces were larger on ions of larger valency.

Note that Cases 7 and 8 considered asymmetry in diffusion coefficient $D_{i}$ but were not shown since the results were identical to those for Case $1\left(D_{1}=D_{2}=9.3 \times 10^{-9} \mathrm{~m}^{2} \mathrm{~s}^{-1}\right)$ everywhere in the electrolyte. Thus, the diffusion coefficient $D_{i}$ had no effect on the concentration profiles for the cycling conditions considered. This was attributed to the fact that galvanostatic cycling was simulated so that the current density $j_{s}$ was imposed and the fluxes of ions into the EDLs remained the same in all cases. Indeed, the increase in cell potential $\psi_{s}(t)$ required to maintain the desired current density $j_{s}$ for electrolytes with smaller diffusion coefficients was negligibly small compared to the potential drop due to the EDLs for Cases 1, 7, and 8.

\subsection{Irreversible Heat Generation Rate $\dot{q}_{i r r}$}

For all Cases $1-8, \dot{q}_{i r r}\left(\right.$ in $\mathrm{W} \mathrm{m}^{-3}$ ) was found to be uniform and equal to $\dot{q}_{i r r}=j_{s}^{2} / \sigma_{\infty}$ throughout the entire diffuse layer of the electrolyte, except in the EDL regions within a few nanometers of the electrode where it decreased steeply to zero (see supplementary material). The differences in $\dot{q}_{i r r}$ summarized in Table 1 reflect the fact that $\sigma_{\infty}$ increased with increasing $\left|z_{i}\right|, D_{i}$, and/or $c_{i, \infty}$ of either ion species, as suggested by Equation (9). Note 
also that, like $\sigma_{\infty}, \dot{q}_{i r r}$ was independent of the ion diameter $a_{i}$, as illustrated by Cases 1,5 , and 6 in Table 1.

\subsection{Reversible Heating}

\subsubsection{Diffusion Heat Generation Rate $\dot{q}_{E, d}$}

Figures $4(\mathrm{a})$ and $4(\mathrm{c})$ show the diffusion heat generation rate $\dot{q}_{E, d}\left(x, 11 t_{c} / 8\right)$ [Equation (11)] at time $t=11 t_{c} / 8$ near the end of a charging step as a function of location $x$ near the positive electrode A for Cases 1-4 and for Cases 1, 5, and 6, respectively. Similarly, Figures 4(b) and $4(\mathrm{~d})$ show $\dot{q}_{E, d}\left(x, 11 t_{c} / 8\right)$ as a function of $2 L-x$ near the negative electrode B for Cases 1-4 and for Cases 1, 5, and 6, respectively. Note that the heat generation rate was plotted at a slightly earlier time than the concentrations shown in Figure 3 in order to show the profiles characteristic of the charging step rather than those during the switch in current direction occurring at $t=3 t_{c} / 2$. Figure 4 indicates that electrolytes with asymmetric valency $z_{i}$ or ion diameter $a_{i}$, e.g., those in Cases 3, 4, and 6, yielded spatially asymmetric heat generation rate $\dot{q}_{E, d}$ profiles. Moreover, for a given electrode, electrolytes sharing the same counterion properties $z_{i}$ and $a_{i}$ as well as bulk concentration $c_{i, \infty}$ yielded the same heat generation rate $\dot{q}_{E, d}$ profiles. In other words, $\dot{q}_{E, d}$ profiles near the positive electrode were identical for Cases 2 and 3 [Figure 4(a)] and for Cases 5 and 6 [Figure 4(c)] while those for Cases 1 and 4 were identical near the negative electrode [Figure 4(b)]. In addition, cases sharing the same counterion properties $z_{i}$ and $a_{i}$, but featuring different bulk concentrations $c_{i, \infty}$ had similar heat generation rate profiles but with different magnitudes. For example, the profile of the heat generation rate $\dot{q}_{E, d}$ for Case 4 spanned the same spatial regions as Case 3 but was larger in magnitude due to the smaller local electrical conductivity $\sigma(x, t)$.

Furthermore, the heat generation rate $\dot{q}_{E, d}$ was confined to a narrower region as the valency $\left|z_{i}\right|$ increased as a result of the narrowing of the EDL region, as discussed previously [Figures 3(a) and 3(b)]. However, the maximum value of $\dot{q}_{E, d}$ remained unaffected by changes in $\left|z_{i}\right|$. This can be attributed to the fact that increasing $\left|z_{i}\right|$ increased the local electrical conductivity $\sigma$ and the concentration gradient by the same proportion in the expression of $\dot{q}_{E, d}$ given by Equation (11). 
Finally, increasing the counterion diameter $a_{i}$ reduced the magnitude of the heat generation rate $\dot{q}_{E, d}$. Although the ion diameter $a_{i}$ does not appear directly in the expression of $\dot{q}_{E, d}$, it affects $\dot{q}_{E, d}$ via its strong influence on the concentration profiles [Figures 3(c) and 3(d)]. Both the concentration gradient and the electrical conductivity $\sigma$ decreased with increasing $a_{i}$. However, the concentration gradient decreased more than the conductivity, resulting in a net decrease in $\dot{q}_{E, d}$. By contrast, changing the diffusion coefficient $D_{i}$ had no effect. In fact, values of $\dot{q}_{E, d}(x, t)$ for Case $7\left(D_{2}=D_{2}=1.1 \times 10^{-9} \mathrm{~m}^{2} \mathrm{~s}^{-1}\right)$ and Case $8\left(D_{1}=1.1 \times 10^{-9} \mathrm{~m}^{2} \mathrm{~s}^{-1}, D_{2}=9.3 \times 10^{-9} \mathrm{~m}^{2} \mathrm{~s}^{-1}\right)$ were identical to those obtained for Case $1\left(D_{2}=D_{2}=9.3 \times 10^{-9} \mathrm{~m}^{2} \mathrm{~s}^{-1}\right)$ at all times and locations. The increase in the diffusion ion fluxes associated with increasing $D_{i}$ was balanced by the corresponding increase in the electrical conductivity $\sigma$. In fact, for electrolytes with symmetric diffusion coefficients $D_{1}=D_{2}=D$, such as Cases 1 and $7, \dot{q}_{E, d}$ was independent of diffusion coefficient $D$ since $D$ appearing in the numerator canceled with that in the expression of $\sigma$ in the denominator [Equation (11)].

\subsubsection{Steric Heat Generation Rate $\dot{q}_{E, s}$}

Figures 5(a) and 5(c) show the steric heat generation rate $\dot{q}_{E, s}\left(x, 11 t_{c} / 8\right)$ [Equation (11)] as a function of location $x$ near the positive electrode A for Cases 1-4 and for Cases 1, 5, and 6 , respectively. Similarly, Figures $5(\mathrm{~b})$ and $5(\mathrm{~d})$ show $\dot{q}_{E, s}\left(x, 11 t_{c} / 8\right)$ as a function of $2 L-x$ near the negative electrode B for Cases 1-4 and for Cases 1, 5, and 6, respectively. Figure 5 indicates that the profiles of the heat generation rates $\dot{q}_{E, s}$ were spatially asymmetric for electrolytes with asymmetric $z_{i}$ and/or $a_{i}$, e.g., those in Cases 3, 4, and 6. It is also interesting to note that the heat generation rate $\dot{q}_{E, s}$ had the same order of magnitude as $\dot{q}_{E, d}$ (Figure 4) for all cases considered. For a given electrode, electrolytes sharing the same counterion properties $z_{i}$ and $a_{i}$ and bulk concentration $c_{i, \infty}$ featured the same heat generation rate $\dot{q}_{E, s}$ profiles. Increasing $\left|z_{i}\right|$ narrowed the region where the steric heat generation rate $\dot{q}_{E, s}$ was significant without affecting its maximum value. However, increasing $a_{i}$ reduced the magnitude of $\dot{q}_{E, s}$. Here also, the steric heat generation rate $\dot{q}_{E, s}$ was unaffected by the diffusion coefficient $D_{i}$. In fact, in the limiting case of $D_{1}=D_{2}=D$, its expression was 
independent of diffusion coefficient.

\subsubsection{Heat of Mixing Heat Generation Rate $\dot{q}_{S, c}$}

Figures $6(\mathrm{a})$ and $6(\mathrm{c})$ show the heat of mixing heat generation rate $\dot{q}_{S, c}\left(x, 11 t_{c} / 8\right)$, [Equation (14)] as a function of location $x$ near the positive electrode A for Cases 1-4 and for Cases 1, 5, and 6, respectively. Similarly, Figures $6(\mathrm{~b})$ and $6(\mathrm{~d})$ show $\dot{q}_{S, c}\left(x, 11 t_{c} / 8\right)$ as a function of $2 L-x$ near the negative electrode B for Cases 1-4 and for Cases 1, 5, and 6, respectively. The heat generation rate profiles $\dot{q}_{S, c}$ near each electrode were very similar for cases sharing the same counterion properties $z_{i}$ and $a_{i}$. The heat of mixing heat generation rate $\dot{q}_{S, c}$ increased strongly with increasing valency $\left|z_{i}\right|$. Indeed, increasing the counterion valency from $\left|z_{i}\right|=1$ to $\left|z_{i}\right|=2$ increased the maximum value of $\dot{q}_{S, c}$ by nearly an order of magnitude. By contrast, recall that $\dot{q}_{E, d}$ and $\dot{q}_{E, s}$ both decreased with increasing $\left|z_{i}\right|$. Equation (14) indicates that, for electrolytes with symmetric valency $-z_{1}=z_{2}=z$ such as in Cases 1 and 2, the local heat generation rate $\dot{q}_{S, c}$ was proportional to $z^{3}$. In fact, for $\left|z_{i}\right|>1, \dot{q}_{S, c}$ provided the largest contribution to the total reversible heat generation rate $\dot{q}_{\text {rev }}$. The strong effect of $\left|z_{i}\right|$ on $\dot{q}_{S, c}$ resulted from strong electrostatic interactions between ions accounted for by the Debye-Hückel activity coefficient [Equation (13)]. Moreover, the heat of mixing heat generation rate $\dot{q}_{S, c}$ decreased with increasing counterion diameter $a_{i}$. Here also, the dependence of $\dot{q}_{S, c}$ on $a_{i}$ was stronger than that of $\dot{q}_{E, d}$ and $\dot{q}_{E, s}$. Finally, results for Cases 7 and 8 with varying $D_{i}$ were identical to those of Case 1 everywhere (not shown). This indicates that $\dot{q}_{S, c}$ was independent of the diffusion coefficient $D_{i}$ as suggested by its definition in Equation (14).

\subsubsection{Overall Reversible Heat Generation Rate $\dot{q}_{\text {rev }}$}

Figures $7(\mathrm{a})$ and $7(\mathrm{c})$ show the reversible heat generation rate $\dot{q}_{r e v}(x, t)=\dot{q}_{E, d}(x, t)+$ $\dot{q}_{E, s}(x, t)+\dot{q}_{S, c}(x, t)+\dot{q}_{S, T}(x, t)$ as a function of location $x$ near the positive electrode A for Cases 1-4 and for Cases 1, 5, and 6, respectively, at time $t=11 t_{c} / 8$ near the end of a charging step. Similarly, Figures $7(\mathrm{~b})$ and $7(\mathrm{~d})$ show $\dot{q}_{r e v}\left(x, 11 t_{c} / 8\right)$ as a function of $2 L-x$ near the negative electrode B for Cases 1-4 and for Cases 1, 5, and 6, respectively. The overall reversible heat generation rate $\dot{q}_{r e v}$ was spatially asymmetric for electrolytes with 
asymmetric $z_{i}$ and $a_{i}$, e.g., those in Cases 3,4 , and 6 . It was larger near the electrode whose counterion had a larger valency $\left|z_{i}\right|$ and/or a smaller ion diameter $a_{i}$. The strong effect of $z_{i}$ on $\dot{q}_{r e v}$ was caused by its effects on $\dot{q}_{S, c}$ which dominated over the other heat generation terms for $\left|z_{i}\right|>1$. Finally, $\dot{q}_{\text {rev }}$ was independent of the diffusion coefficient $D_{i}$ for all cases considered. Note that the heat generation rate $\dot{q}_{S, T}$ [Equation (14)] was negligible compared with the other heat generation rates for Cases 1-8. This was also observed in our previous study for binary and symmetric electrolytes [8].

\subsubsection{Total Heat Generation Rates $\dot{Q}_{i r r}^{\prime \prime}$ and $\dot{Q}_{r e v}^{\prime \prime}$}

It is difficult to directly compare the total irreversible and reversible heating based on the local volumetric heat generation rates $\dot{q}_{i r r}$ and $\dot{q}_{r e v}$. Indeed, in all cases, $\dot{q}_{i r r}$ was very small compared to the peak values of $\dot{q}_{r e v}$. However, $\dot{q}_{i r r}$ was uniform throughout the $40 \mu$ m-thick electrolyte region while $\dot{q}_{\text {rev }}$ was significant only inside the EDL region located within a few nanometers of the electrode surfaces. To compare their relative significance, they should be integrated over the entire electrolyte region to assess their overall contribution to the total heat generation. To do so, the total irreversible and reversible heat generation rates per unit separator surface area (in $\mathrm{W} \mathrm{m}^{-2}$ ) were defined as

$$
\dot{Q}_{i r r}^{\prime \prime}(t)=\int_{0}^{2 L} \dot{q}_{i r r}(x, t) d x \quad \text { and } \quad \dot{Q}_{r e v}^{\prime \prime}(t)=\int_{0}^{2 L} \dot{q}_{r e v}(x, t) d x .
$$

Figure 8 shows the total reversible heat generation rate $\dot{Q}_{r e v}^{\prime \prime}(t)$ as a function of time $t$ for (a) Cases 1-4 with different values of $z_{i}$ and (b) Cases 1, 5, and 6 with different values of $a_{i}$ over one and a half cycles. The corresponding values of $\dot{Q}_{i r r}^{\prime \prime}(t)$ were constant and equal to $\dot{Q}_{i r r}^{\prime \prime}=2 \dot{q}_{i r r} L$ where $L=20 \mu \mathrm{m}$ and $\dot{q}_{i r r}$ is reported in Table 1 . The total reversible heat generation rate $\dot{Q}_{r e v}^{\prime \prime}(t)$ was periodic in time $t$, positive during charging, and negative during discharging. For the conditions simulated, the reversible heat generation rate $\dot{Q}_{r e v}^{\prime \prime}(t)$ was systematically larger than $\dot{Q}_{i r r}^{\prime \prime}$. Indeed, the maximum value of $\left|\dot{Q}_{r e v}^{\prime \prime}(t)\right|$ was on the order of 20 to $40 \mathrm{~W} \mathrm{~m}^{-2}$ while $\dot{Q}_{i r r}^{\prime \prime}$ was on the order of 0.001 to $0.1 \mathrm{~W} \mathrm{~m}^{-2}$. Note that previous simulations for binary and symmetric organic electrolyte, namely $\mathrm{TEABF}_{4}$ in propylene carbonate, also predicted that $\left|\dot{Q}_{r e v}^{\prime \prime}(t)\right|$ was larger than $\dot{Q}_{i r r}^{\prime \prime}(t)$ by about one 
order of magnitude [8]. The relatively small values of $\dot{Q}_{i r r}^{\prime \prime}(t)$ obtained in the present study compared to that in Ref. [8] can be attributed to the larger conductivity $\sigma_{\infty}$ of the electrolyte resulting in smaller Joule heating.

Figure 8(a) establishes that increasing either or both ion valencies $\left|z_{1}\right|$ and $\left|z_{2}\right|$ led to larger reversible heat generation while Joule heating decreased (Table 1). Cases 1-3 corresponded to the same bulk electrolyte concentration $c_{1, \infty}$. Comparing these cases reveals that $\dot{Q}_{r e v}^{\prime \prime}(t)$ and $\dot{Q}_{i r r}^{\prime \prime}(t)$ for Case $3\left(z_{1}=-2, z_{2}=1\right)$ with asymmetric $z_{i}$ fell between those corresponding to symmetric electrolytes, namely Case $1\left(-z_{1}=z_{2}=1\right)$ and Case $2\left(-z_{1}=z_{2}=2\right)$. Cases 3 and 4 demonstrate that the total heat generation rates were also sensitive to the bulk ion concentrations $c_{i, \infty}$. These two cases featured the same ion properties $z_{i}, a_{i}$, and $D_{i}$ for both ion species, but the bulk ion concentrations $c_{i, \infty}$ of Case 4 were half those of Case 3. The results established that both $\left|\dot{Q}_{r e v}^{\prime \prime}(t)\right|$ and $\dot{Q}_{i r r}^{\prime \prime}(t)$ increased with decreasing bulk concentrations $c_{1, \infty}$ and $c_{2, \infty}$ due to the associated smaller electrical conductivity $\sigma_{\infty}$.

Figure $8(\mathrm{~b})$ shows the total reversible heat generation rate $\dot{Q}_{r e v}^{\prime \prime}(t)$ as a function of time $t$ over one and a half cycles for Cases 1,5 , and 6 corresponding to different values of $a_{1}$ and $a_{2}$. The total irreversible heat generation rate $\dot{Q}_{i r r}^{\prime \prime}(t)$ was unaffected by $a_{i}$ and remained constant and equal to $\dot{Q}_{i r r}^{\prime \prime}=11 \mathrm{~mW} \mathrm{~m}^{-2}$. It was smaller than $\left|\dot{Q}_{r e v}^{\prime \prime}(t)\right|$ by several orders of magnitude. On the other hand, the total reversible heat generation rate $\left|\dot{Q}_{\text {rev }}^{\prime \prime}(t)\right|$ increased with decreasing $a_{i}$ for either ion species. For Case 6 with asymmetric $a_{i}\left(a_{1}=0.76 \mathrm{~nm}\right.$, $\left.a_{2}=0.56 \mathrm{~nm}\right), \dot{Q}_{r e v}^{\prime \prime}(t)$ was approximately equal to the arithmetic mean of those for the corresponding symmetric electrolytes represented by Case $1\left(a_{1}=a_{2}=0.56 \mathrm{~nm}\right)$ and Case $5\left(a_{1}=a_{2}=0.76 \mathrm{~nm}\right)$.

Finally, the total irreversible heat generation rate $\dot{Q}_{i r r}^{\prime \prime}(t)$ for Case $1\left(D_{1}=D_{2}=9.3 \times 10^{-9} \mathrm{~m}^{2} \mathrm{~s}^{-1}\right)$, Case $7\left(D_{1}=D_{2}=1.1 \times 10^{-9} \mathrm{~m}^{2} \mathrm{~s}^{-1}\right)$, and Case $8\left(D_{1}=1.1 \times 10^{-9} \mathrm{~m}^{2} \mathrm{~s}^{-1}, D_{2}=9.3 \times 10^{-9} \mathrm{~m}^{2} \mathrm{~s}^{-1}\right)$ featuring different values of $D_{i}$ was constant and equal to $11 \mathrm{~mW} \mathrm{~m}^{-2}, 95 \mathrm{~mW} \mathrm{~m}^{-2}$, and $20 \mathrm{~mW} \mathrm{~m}^{-2}$, respectively. Its magnitude increased with decreasing diffusion coefficient $D_{i}$ of either ion. It was more strongly influenced by the larger of the two diffusion coefficients. This can be attributed to the fact that the ion with the larger diffusion coefficient carried 
the majority of the current density within the bulk electrolyte. On the other hand, $\dot{Q}_{r e v}^{\prime \prime}(t)$, like the local reversible heat generation rates $\dot{q}_{E, d}, \dot{q}_{E, s}$, and $\dot{q}_{S, c}$, was unaffected by the values of $D_{i}$ and equal to that of the baseline Case 1 (Figure 8).

\subsection{Temperature}

Figure 9 shows the temperature differences (a) $T(a / 2, t)-T_{0}$ near the positive electrode A, (b) $T(L, t)-T_{0}$ at the centerline, and (c) $T(2 L-a / 2, t)-T_{0}$ near the negative electrode $\mathrm{B}$ for Cases 1-4 featuring different values of $z_{i}$. Note that the temperature rise for the present simulations was on the order of millikelvins. This can be attributed to the very rapid charging of planar electrodes resulting in very small cycle periods on the order of milliseconds. Cases 3 and 4 had asymmetric valency $z_{1}=-2$ and $z_{2}=1$ and yielded spatially asymmetric temperature oscillations caused by the asymmetric reversible heat generation rate $\dot{q}_{r e v}$. In both cases, the temperature oscillations near the positive electrode [Figure 9(a)] were approximately three times larger than as those near the negative electrode [Figure 9(c)]. This can be attributed to the fact that the large valency $z_{1}=-2$ of the anion produced large $\dot{q}_{S, c}$ near the positive electrode. In addition, the asymmetric electrolyte considered in Cases 3 and 4 produced larger temperature oscillations at $x=a / 2$ than either of the symmetric electrolytes of Case $1\left(-z_{1}=z_{2}=1\right)$ and Case $2\left(-z_{1}=z_{2}=2\right)$. For symmetric electrolytes as in Cases 1 and 2, the temperature profiles always remained spatially symmetric so that no significant heat fluxes crossed the cell centerline. By contrast, for asymmetric electrolytes as in Cases 3 and 4, there was significant heat exchange between the two halves of the cell. As a result, the temperatures evolved differently between the symmetric and asymmetric electrolytes.

Figures $9(\mathrm{~d}), 9(\mathrm{e})$, and $9(\mathrm{f})$ respectively show the temperature difference $T(a / 2, t)-T_{0}$ near the positive electrode A, $T(L, t)-T_{0}$ at the centerline, and $T(2 L-a / 2, t)-T_{0}$ near the negative electrode $\mathrm{B}$ for Cases 1, 5, and 6 featuring different values of $a_{i}$. The asymmetric electrolyte of Case $6\left(a_{1}=0.76 \mathrm{~nm}, a_{2}=0.56 \mathrm{~nm}\right)$ exhibited larger temperature oscillations near the negative electrode [Figure 9(f)] than near the positive electrode [Figure 9(d)]. This can be attributed to the larger reversible heat generation rate $\dot{q}_{r e v}$ associated with the smaller 
cation diameter $a_{2}=0.56 \mathrm{~nm}$.

Moreover, Figures 9(b) and 9(e) indicate that, for a given case, the temperature oscillations at the centerline were smaller in amplitude, less angular, and slightly delayed compared to those close to the electrodes. This was also observed for binary and symmetric electrolytes. It was attributed to the fact that the reversible heat generated at the electrode surfaces had to conduct through the electrolyte to reach the centerline [8]. In addition, the centerline temperature $T(L, t)$ for the asymmetric electrolyte of Case 3 $\left(z_{1}=-2, z_{2}=1\right)$ fell between those of the symmetric electrolytes considered in Case 1 $\left(-z_{1}=z_{2}=1\right)$ and Case $2\left(-z_{1}=z_{2}=2\right)$ corresponding to the same bulk concentration $c_{1, \infty}$. Likewise, the centerline temperature $T(L, t)[$ Figure $9(\mathrm{e})]$ for the asymmetric electrolyte of Case $6\left(a_{1}=0.76 \mathrm{~nm}, a_{2}=0.56 \mathrm{~nm}\right)$ fell between those of the corresponding symmetric electrolytes represented by Case $1\left(a_{1}=a_{2}=0.56 \mathrm{~nm}\right)$ and Case $5\left(a_{1}=a_{2}=0.76 \mathrm{~nm}\right)$. Note also that the centerline temperature $T(L, t)$ for Cases 1,5 , and 6 converged to the same value after each complete cycle (e.g., $t=7.6 \mathrm{~ms})$. Indeed, reversible heating during the charging step was compensated by reversible cooling during the discharging step. Thus, the net reversible heat generated over a complete charge-discharge cycle was $\int_{0}^{t_{c}} \dot{Q}_{r e v}^{\prime \prime}(t) d t=0$ and the net temperature rise over one cycle was solely due to the irreversible heat generation rate $\dot{Q}_{i r r}^{\prime \prime}$. For these three cases, $\dot{Q}_{i r r}^{\prime \prime}$ was identical (Table 1$)$.

Table 2 summarizes qualitative changes in the heat generation rates $\dot{q}_{i r r}, \dot{q}_{r e v}, \dot{Q}_{i r r}^{\prime \prime}$, and $\dot{Q}_{r e v}^{\prime \prime}$ as well as in the integral areal capacitance $C_{s, i n t}$ caused by increasing the ions' effective diameter $a_{i}$, valency $\left|z_{i}\right|$, diffusion coefficient $D_{i}$, or bulk concentration $c_{i, \infty}$. Note that the bulk concentrations $c_{1, \infty}$ and $c_{2, \infty}$ cannot be varied independently, as they are coupled by the electroneutrality requirement. Table 2 suggests a few design rules for choosing electrolytes in order to minimize heat generation in EDLCs. First, large bulk concentrations $c_{i, \infty}$ are desirable to increase ionic conductivity $\sigma$ and thus reduce both the total irreversible and reversible heat generation rates $\dot{Q}_{i r r}^{\prime \prime}$ and $\dot{Q}_{r e v}^{\prime \prime}$ as well as increasing the capacitance. Similarly, at least one ion with large diffusion coefficient $D_{i}$ should be used to minimize $\dot{Q}_{i r r}^{\prime \prime}$. This ion species will carry the majority of the current in the bulk electrolyte. Note that large diffu- 
sion coefficient is also beneficial to the EDLC capacitance at large scan rates [45]. Finally, increasing $\left|z_{i}\right|$ and/or decreasing $a_{i}$ increases the EDLC capacitance $C_{s, \text { int }}$ but also increases the reversible heat generation rate $\dot{Q}_{r e v}^{\prime \prime}$. In particular, thermal management strategies for EDLCs using large-valency ions should be designed to accommodate large reversible heat generation rates $\dot{q}_{r e v}$ near the counter-electrode.

\subsection{Asymmetric Electrolytes in Porous Electrodes}

The present simulations were limited to planar electrodes while practical EDLC devices use porous electrodes. The present model could be extended to porous electrodes in at least three possible ways. First, the continuum model presented in Section 3.2 could be used to predict local heat generation rates and temperature in transient two-dimensional or three-dimensional simulations of porous electrodes. However, such simulations would be computationally very costly and time consuming and fall beyond the scope of the present study. Second, volume averaging theory could be applied to the governing equations derived in the present study, as performed for numerous transport phenomena in porous media [46]. Third, correlations predicting the heat generation rates in porous electrodes could be developed based on scaling analysis of the governing equations for planar electrodes modified by a semi-empirical geometric parameter identified from experimental data for porous electrodes. This method was successfully used to develop a correlation for the equilibrium areal capacitance of porous electrodes in Ref. [47]. We anticipate that the qualitative observations regarding the effects of ion valency and size reported for planar electrodes will also apply to porous electrodes regardless of their morphology.

Finally, it is important to note that the electrode's porous morphology adds complications that cannot be accounted for in the planar analysis. First, when the effective ion diameter is too large for ions to enter the pores, the active electrode surface area is reduced and consequently the overall reversible heating would be reduced. In addition, ions may shed their solvation shells to enter pores smaller than their solvated radii, resulting in a sharp increase in capacitance $[48,49]$. However, the present model assumes that the effective diameter of the ions is constant. As a result, it cannot predict the thermal effects associated with ion 
desolvation.

\section{Conclusion}

The present study developed the first thermal model based on first principles for the local irreversible and reversible heat generation rates and temperature of EDLCs with multiple ion species and/or asymmetric electrolytes. Detailed numerical simulations were performed for different binary and asymmetric electrolytes based on the properties of aqueous $\mathrm{H}_{2} \mathrm{SO}_{4}$. First, the irreversible heat generation rate $\dot{q}_{i r r}$ was uniform across the electrolyte and equal to $\dot{q}_{i r r}=$ $j_{s}^{2} / \sigma_{\infty}$. It decreased with increasing valency $\left|z_{i}\right|$ or diffusion coefficient $D_{i}$ of one or both ion species due to the resulting increase in electrical conductivity of the electrolyte. However,

$\dot{q}_{i r r}$ was independent of the ion diameter $a_{i}$. The reversible heat generation rate $\dot{q}_{r e v}$ near each electrode was governed by the properties of the counterion. It increased with increasing valency $\left|z_{i}\right|$ and decreasing ion diameter $a_{i}$ but was independent of diffusion coefficient $D_{i}$. As a result, electrolytes with asymmetric valency $z_{i}$ or ion diameter $a_{i}$ featured spatially asymmetric heat generation rates and larger temperature oscillations near the electrode with the larger $\left|z_{i}\right|$ or smaller $a_{i}$ of the counterion. The results demonstrate that thermal models must account for electrolyte asymmetry in order to accurately predict the local heat generation rates and temperature. This study suggests that to reduce the overall heat generation in EDLCs, electrolytes should feature large bulk concentrations $c_{i, \infty}$ and at least one ion species with large diffusion coefficient. In addition, electrolytes chosen to yield large capacitance via ions with large valency $\left|z_{i}\right|$ and/or small diameter $a_{i}$ are likely to feature large reversible heat generation rates generated near the electrode surfaces.

\section{Acknowledgement}

This material is based upon work supported by the National Science Foundation Graduate Research Fellowship under Grant No. DGE-1144087 and as part of the Molecularly Engineered Energy Materials, an Energy Frontier Research Center funded by the U.S. Department of Energy, Office of Science, Office of Basic Energy Sciences under Award Number DE-SC0001342. 


\section{References}

\section{References}

[1] B. Conway, Electrochemical Supercapacitors: Scientific Fundamentals and Technological Applications, Kluwer Academic/Plenum Publishers, New York, NY, 1999.

[2] US Department of Energy, Basic Research Needs for Electrical Energy Storage: Report of the Basic Energy Sciences Workshop for Electrical Energy Storage, Tech. rep., Office of Basic Energy Sciences, DOE (2007).

URL http://www.osti.gov/accomplishments/documents/fullText/ACC0330.pdf

[3] T. Pandolfo, V. Ruiz, S. Sivakkumar, J. Nerkar, Supercapacitors: Materials, Systems, and Applications, Wiley-VCH Verlag, Weinheim, Germany, 2013, Ch. 2: General properties of electrochemical capacitors, pp. 69-109.

[4] J. R. Miller, Electrochemical capacitor thermal management issues at high-rate cycling, Electrochimica Acta 52 (4) (2006) 1703-1708.

[5] J. Schiffer, D. Linzen, D. U. Sauer, Heat generation in double layer capacitors, Journal of Power Sources 160 (1) (2006) 765-772.

[6] P. Guillemet, Y. Scudeller, T. Brousse, Multi-level reduced-order thermal modeling of electrochemical capacitors, Journal of Power Sources 157 (1) (2006) 630-640.

[7] H. Gualous, H. Louahlia-Gualous, R. Gallay, A. Miraoui, Supercapacitor thermal modeling and characterization in transient state for industrial applications, IEEE Transactions on Industry Applications 45 (3) (2009) 1035-1044.

[8] A. L. d'Entremont, L. Pilon, First-principles thermal modeling of electric double layer capacitors under constant-current cycling, Journal of Power Sources 246 (2014) 887 898. 
[9] M. Al Sakka, H. Gualous, J. Van Mierlo, H. Culcu, Thermal modeling and heat management of supercapacitor modules for vehicle applications, Journal of Power Sources 194 (2) (2009) 581-587.

[10] O. Bohlen, J. Kowal, D. U. Sauer, Ageing behaviour of electrochemical double layer capacitors: Part II. Lifetime simulation model for dynamic applications, Journal of Power Sources 173 (1) (2007) 626-632.

[11] H. Gualous, H. Louahlia, R. Gallay, Supercapacitor characterization and thermal modelling with reversible and irreversible heat effect, IEEE Transactions on Power Electronics 26 (11) (2011) 3402-3409.

[12] C.-J. Chiang, J.-L. Yang, W.-C. Cheng, Temperature and state-of-charge estimation in ultracapacitors based on extended Kalman filter, Journal of Power Sources 234 (2013) $234-243$.

[13] H. Wang, A. Thiele, L. Pilon, Simulations of cyclic voltammetry for electric double layers in asymmetric electrolytes: A generalized modified Poisson-Nernst-Planck model, Journal of Physical Chemistry C 117 (2013) 18286-18297.

[14] W. Haynes, T. J. Bruno, D. R. Lide (Eds.), CRC Handbook of Chemistry and Physics, 94th Edition, CRC Press, Boca Raton, FL, 2013.

[15] J. N. Israelachvili, Intermolecular and Surface Forces, 2nd Edition, Academic Press, London, UK, 1991.

[16] A. Horvath, Handbook of Aqueous Electrolyte Solutions: Physical Properties, Estimation and Correlation Methods, Ellis Horwood Ltd, Chichester, UK, 1985.

[17] R. Lin, P.-L. Taberna, S. Fantini, V. Presser, C. R. Pérez, F. Malbosc, N. L. Rupesinghe, K. B. K. Teo, Y. Gogotsi, P. Simon, Capacitive energy storage from -50 to $100^{\circ} \mathrm{C}$ using an ionic liquid electrolyte, The Journal of Physical Chemistry Letters 2 (2011) 23962401. 
[18] S. Li, G. Feng, P. F. Fulvio, P. C. Hillesheim, C. Liao, D. Shang, Molecular dynamics simulation study of the capacitive performance of a binary mixture of ionic liquids near an onion-like carbon electrode, The Journal of Physical Chemistry Letters 3 (2012) 2465-2469.

[19] R. Palm, H. Kurig, K. Tõnurist, A. Jänes, E. Lust, Is the mixture of 1-ethyl-3methylimidazolium tetrafluoroborate and 1-butyl-3-methylimidazolium tetrafluoroborate applicable as electrolyte in electrical double layer capacitors?, Electrochemistry Communications 22 (2012) $203-206$.

[20] A. Bard, L. Faulkner, Electrochemical Methods: Fundamentals and Applications, John Wiley \& Sons, New York, NY, 2001.

[21] V. Bagotsky, Fundamentals of Electrochemistry, John Wiley \& Sons, Hoboken, NJ, 2006.

[22] J. S. Newman, K. E. Thomas-Alyea, Electrochemical Systems, 3rd Edition, John Wiley \& Sons, Hoboken, NJ, 2004.

[23] M. S. Kilic, M. Z. Bazant, A. Ajdari, Steric effects in the dynamics of electrolytes at large applied voltages. II. Modified Poisson-Nernst-Planck equations, Physical Review E 75 (2) (2007) 021503.

[24] H. Wang, L. Pilon, Reply to comments on "Intrinsic limitations of impedance measurements in determining electric double layer capacitances" by H. Wang, L. Pilon [Electrochimica Acta 63 (2012) 55], Electrochemica Acta 76 (2012) 529-531.

[25] M. S. Kilic, M. Z. Bazant, A. Ajdari, Steric effects in the dynamics of electrolytes at large applied voltages. I. Double-layer charging, Physical Review E 75 (2007) 021502.

[26] J. Baker-Jarvis, B. Riddle, Ion dynamics near charged electrodes with excluded volume effect, IEEE Transactions on Dielectrics and Electrical Insulation 6 (2) (1999) 226-235. 
[27] J. Cervera, V. García-Morales, J. Pellicer, Ion size effects on the electrokinetric flow in nanoporous membranes caused by concentration gradients, Journal of Physical Chemistry B 107 (2003) 8300-8309.

[28] K. T. Chu, M. Z. Bazant, Surface conservation laws at microscopically diffuse interfaces, Journal of Colloid and Interface Science 315 (2007) 319-329.

[29] M. Z. Bazant, M. S. Kilic, B. Storey, A. Ajdari, Towards an understanding of inducedcharge electrokinetics at large applied volages in concentrated solutions, Advances in Colloid and Interfacial Science 152 (1-2) (2009) 48-88.

[30] M. Aranda-Rascón, C. Grosse, J. López-García, J. Horno, Influence of the finite ion size on the predictions of the standard electrokinetic model: Frequency response, Journal of Colloid and Interface Science 336 (2) (2009) 857-864.

[31] L. H. Olesen, M. Z. Bazant, H. Bruus, Strongly nonlinear dynamics of electrolytes in large ac voltages, Physical Review E 82 (2010) 011501.

[32] J. Cervera, P. Ramírez, J. A. Manzanares, S. Mafé, Incorporating ionic size in the transport equations for charged nanopores, Microfluidics and Nanofluidics 9 (2010) 4153.

[33] H. Zhao, Diffuse-charge dynamics of ionic liquids in electrochemical systems, Physical Review E 84 (2011) 051504.

[34] Z. Adamczyk, P. Warszyński, Role of electrostatic interactions in particle adsorption, Advances in Colloid and Interface Science 63 (1996) 41-149.

[35] R. F. Probstein, Physicochemical Hydrodynamics: An Introduction, 2nd Edition, John Wiley \& Sons, Hoboken, NJ, 2003.

[36] R. B. Bird, W. E. Stewart, E. N. Lightfoot, Transport Phenomena, John Wiley \& Sons, New York, NY, 1962. 
[37] W. Gu, C. Wang, Thermal-electrochemical modeling of battery systems, Journal of the Electrochemical Society 147 (8) (2000) 2910-2922.

[38] P. Biesheuvel, D. Brogioli, H. Hamelers, Negative Joule heating in ion-exchange membranes, arXiv:1402.1448v1 (2014).

[39] J. Joule, On the heat evolved by metallic conductors of electricity, and in the cells of a battery during electrolysis, Philosophical Magazine 19 (1841) 260-277.

[40] B. J. Kirby, Micro- and Nanoscale Fluid Mechanics: Transport in Microfluidic Devices, Cambridge University Press, New York, NY, 2010.

[41] P. Debye, E. Hückel, Zur Theorie der Elektrolyte. I. Gefrierpunktserniedrigung und verwandte Erscheinungen (On the theory of electrolytes. I. Freezing point depression and related phenomena), Physikalische Zeitschrift 24 (1923) 185-206.

[42] COMSOL, Inc., COMSOL Multiphysics Reference Manual, COMSOL 4.4 Edition (2013).

[43] Y. Dandeville, P. Guillemet, Y. Scudeller, O. Crosnier, L. Athouel, T. Brousse, Measuring time-dependent heat profiles of aqueous electrochemical capacitors under cycling, Thermochimica Acta 526 (2011) 1-8.

[44] C. Pascot, Y. Dandeville, Y. Scudeller, P. Guillemet, T. Brousse, Calorimetric measurement of the heat generated by a double-layer capacitor cell under cycling, Thermochimica Acta 510 (2010) 53-60.

[45] H. Wang, L. Pilon, Physical interpretation of cyclic voltammetry for measuring electric double layer capacitances, Electrochimica Acta 64 (2012) 130-139.

[46] S. Whitaker, The Method of Volume Averaging, Kluwer Academic Press, Dordrecht, the Netherlands, 2010. 
[47] H. Wang, J. Fang, L. Pilon, Scaling laws for carbon-based electric double layer capacitors, Electrochimica Acta 109 (2013) 316-321.

[48] J. Chmiola, G. Yushin, Y. Gogotsi, C. Portet, P. Simon, P. Taberna, Anomalous increase in carbon capacitance at pore sizes less than 1 nanometer, Science 313 (5794) (2006) $1760-1763$.

[49] P. Simon, Y. Gogotsi, Materials for electrochemical capacitors, Nature Materials 7 (2008) 845-854. 
(a)

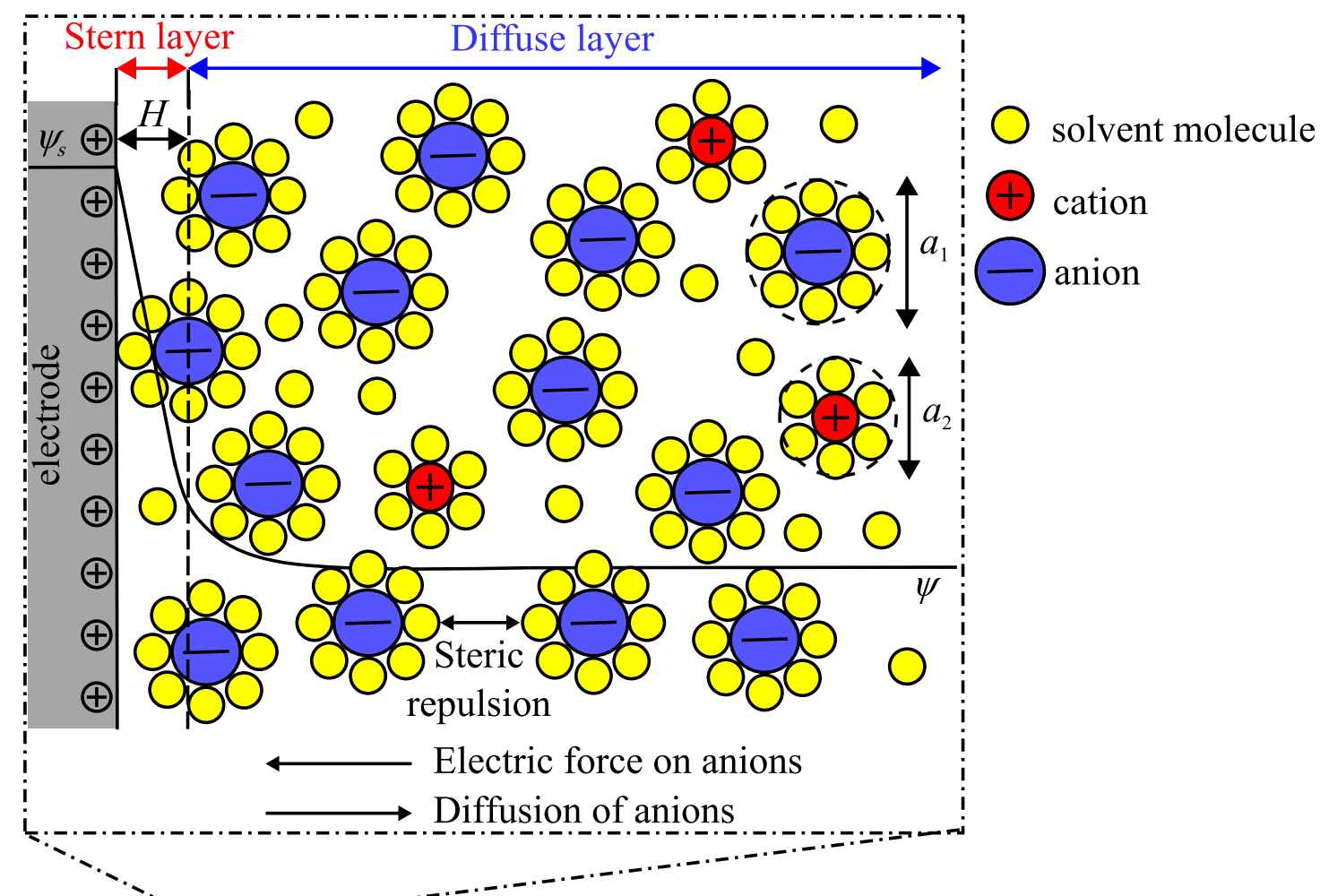

(b)

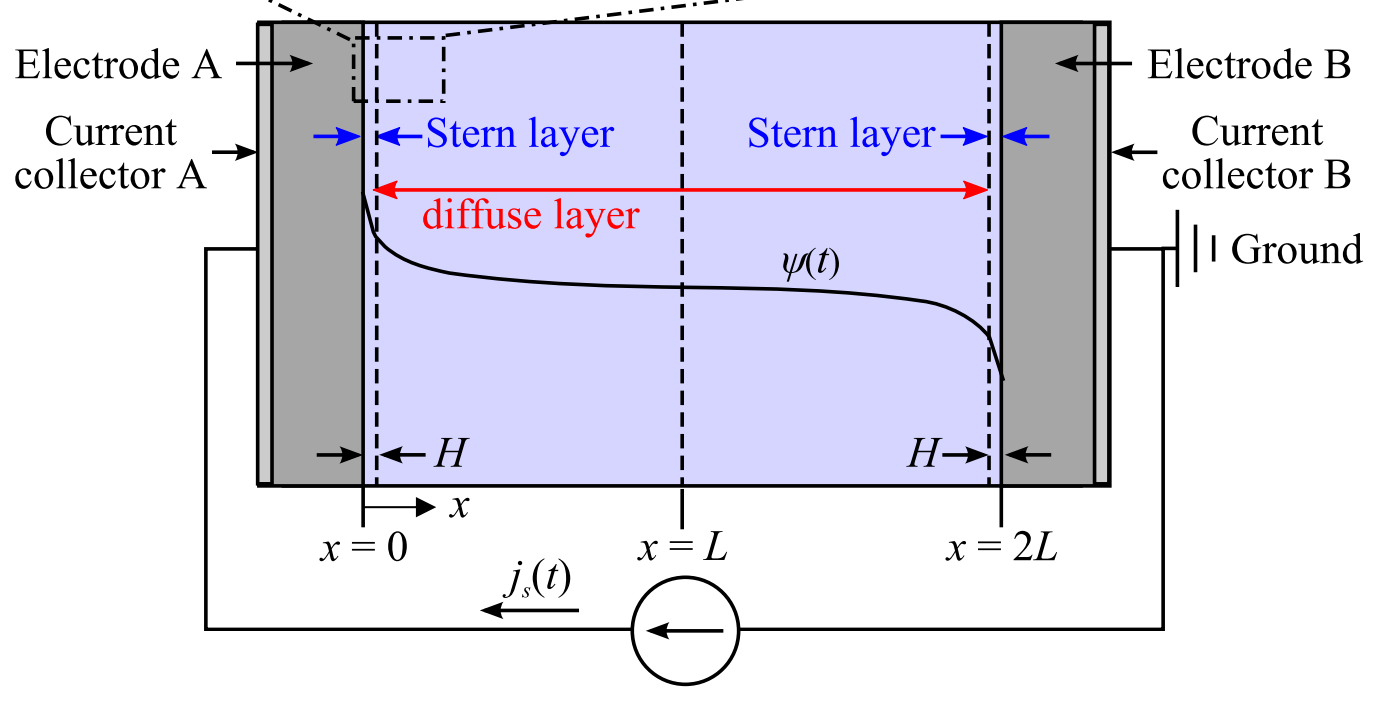

Figure 1: Illustration of (a) the basic structure of an EDLC with planar electrodes and (b) the electric double layer structure of a binary and asymmetric electrolyte near a planar electrode. 

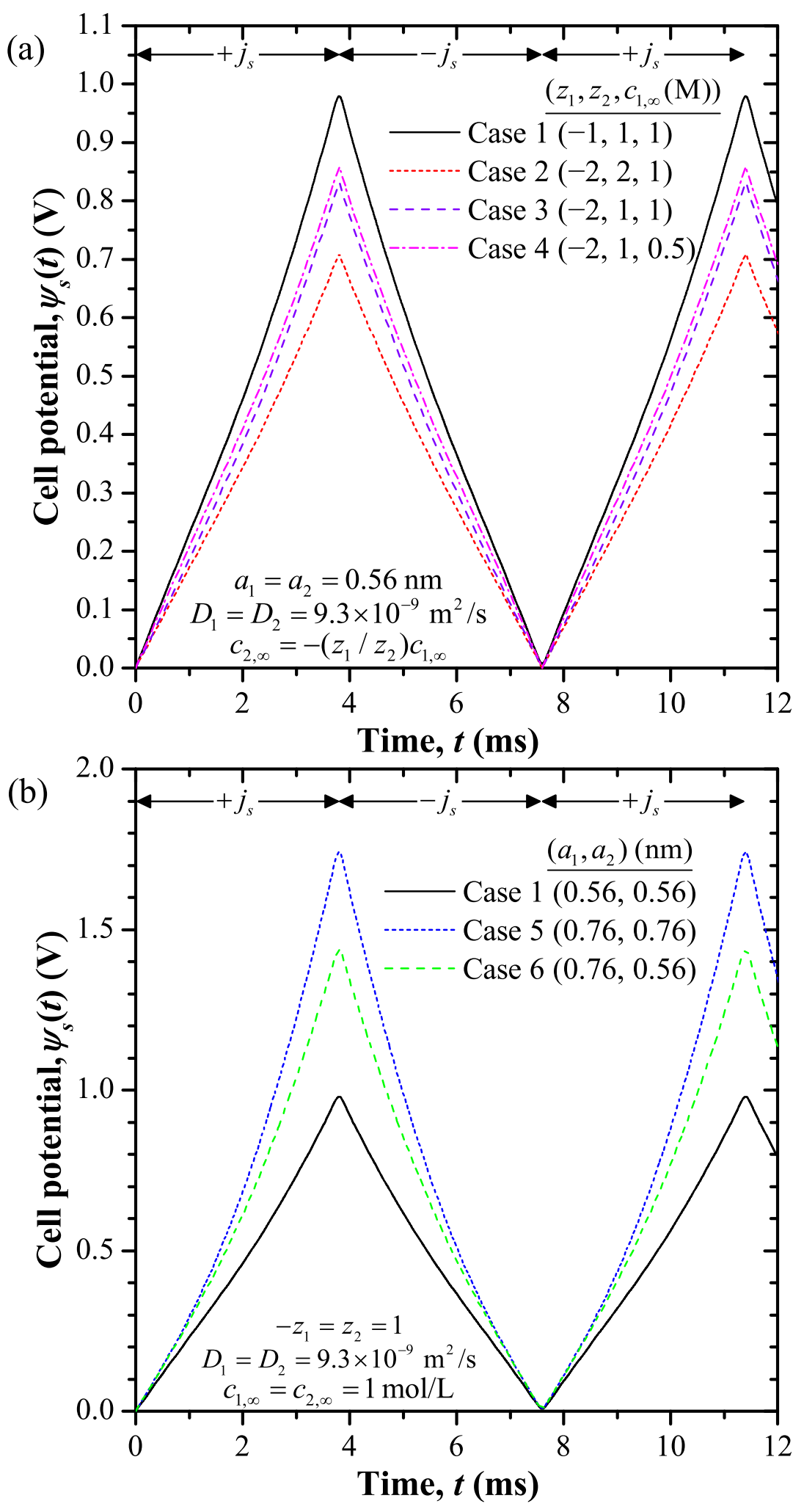

Figure 2: Electric potential difference across the EDLC $\psi_{s}(t)=\psi(0, t)-\psi(2 L, t)$ as a function of time $t$ over the first cycle and a half for (a) Cases 1-4 featuring different ion valencies $z_{i}$ and (b) Cases 1, 5, and 6 featuring different effective ion diameters. Results for Cases 7 and 8 featuring different diffusion coefficients $D_{i}$ were identical to those of Case 1. 
Effect of valency $\left|z_{i}\right|$

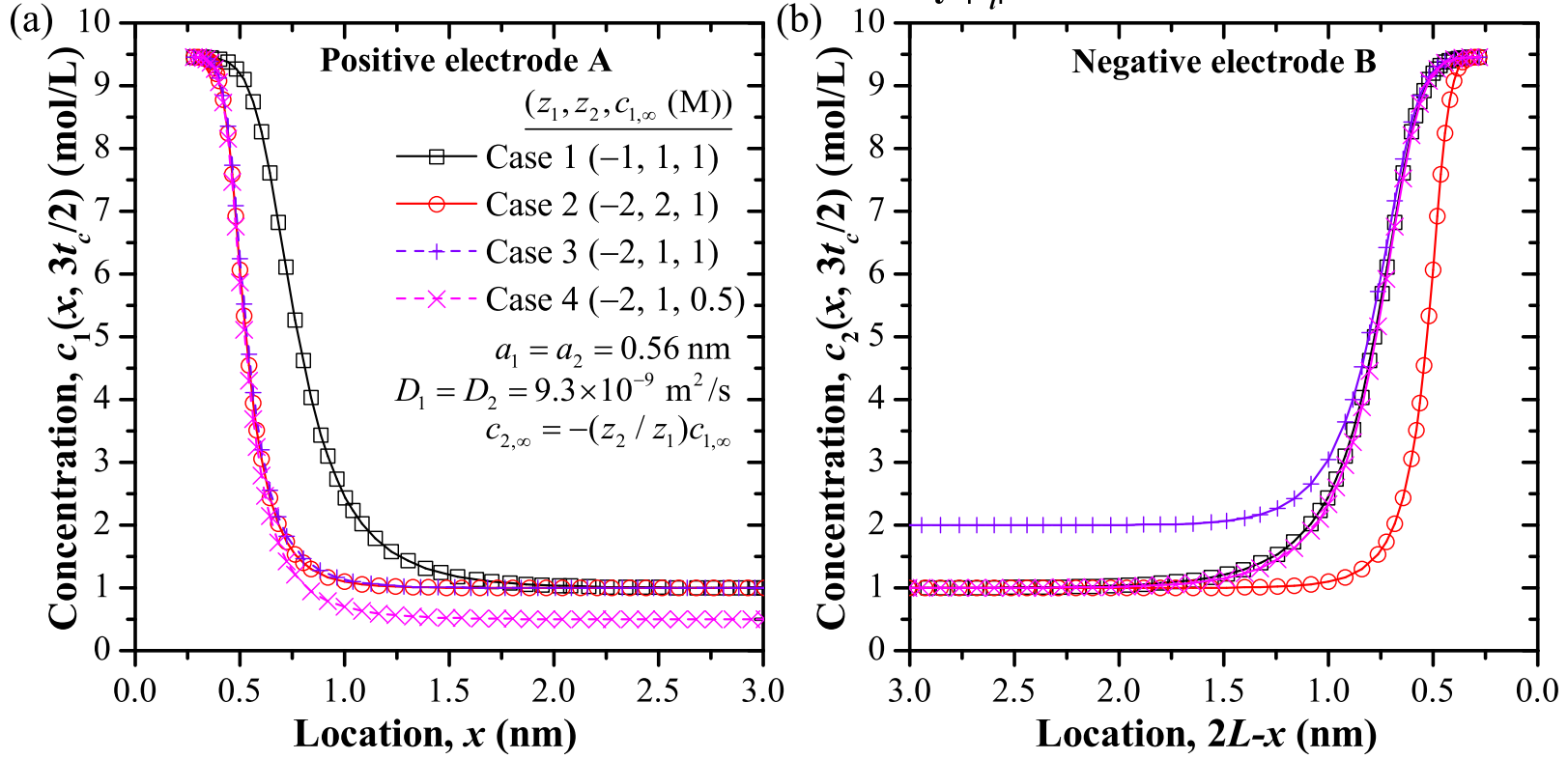

Effect of ion diameter $\boldsymbol{a}_{i}$
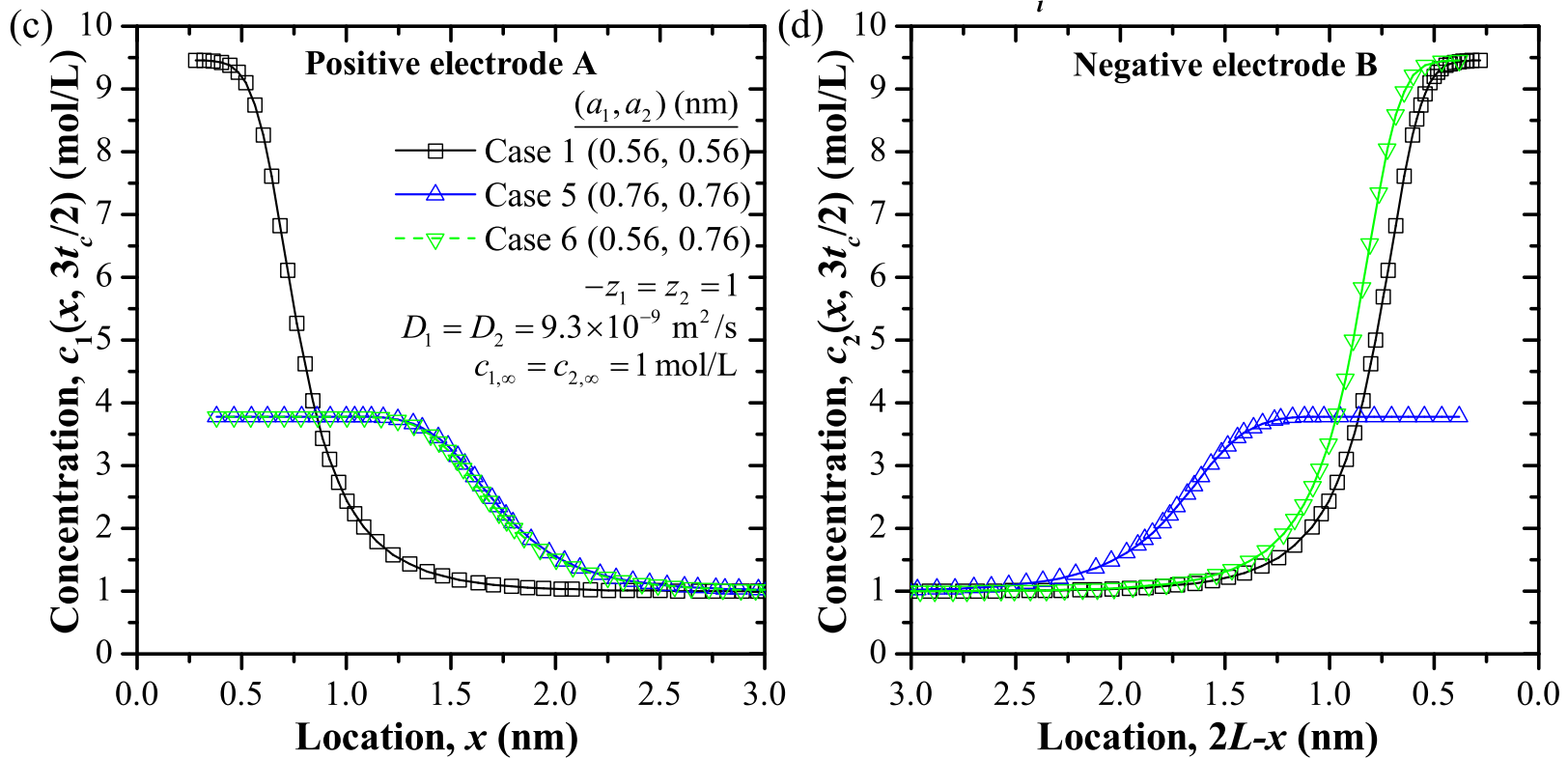

Figure 3: Anion concentration $c_{1}\left(x, 3 t_{c} / 2\right)$ near the positive electrode $\mathrm{A}$ as a function of location $x$ for (a) Cases 1-4 with different values of $z_{i}$ and (c) Cases 1, 5, and 6 with different values of $a_{i}$ as well as cation concentration $c_{2}\left(x, 3 t_{c} / 2\right)$ near the negative electrode $\mathrm{B}$ as a function of location $2 L-x$ for (b) Cases 1-4 and (d) Cases 1, 5, and 6. All cases correspond to time $t=3 t_{c} / 2$ at the end of a charging step. 
Effect of valency $\left|z_{i}\right|$
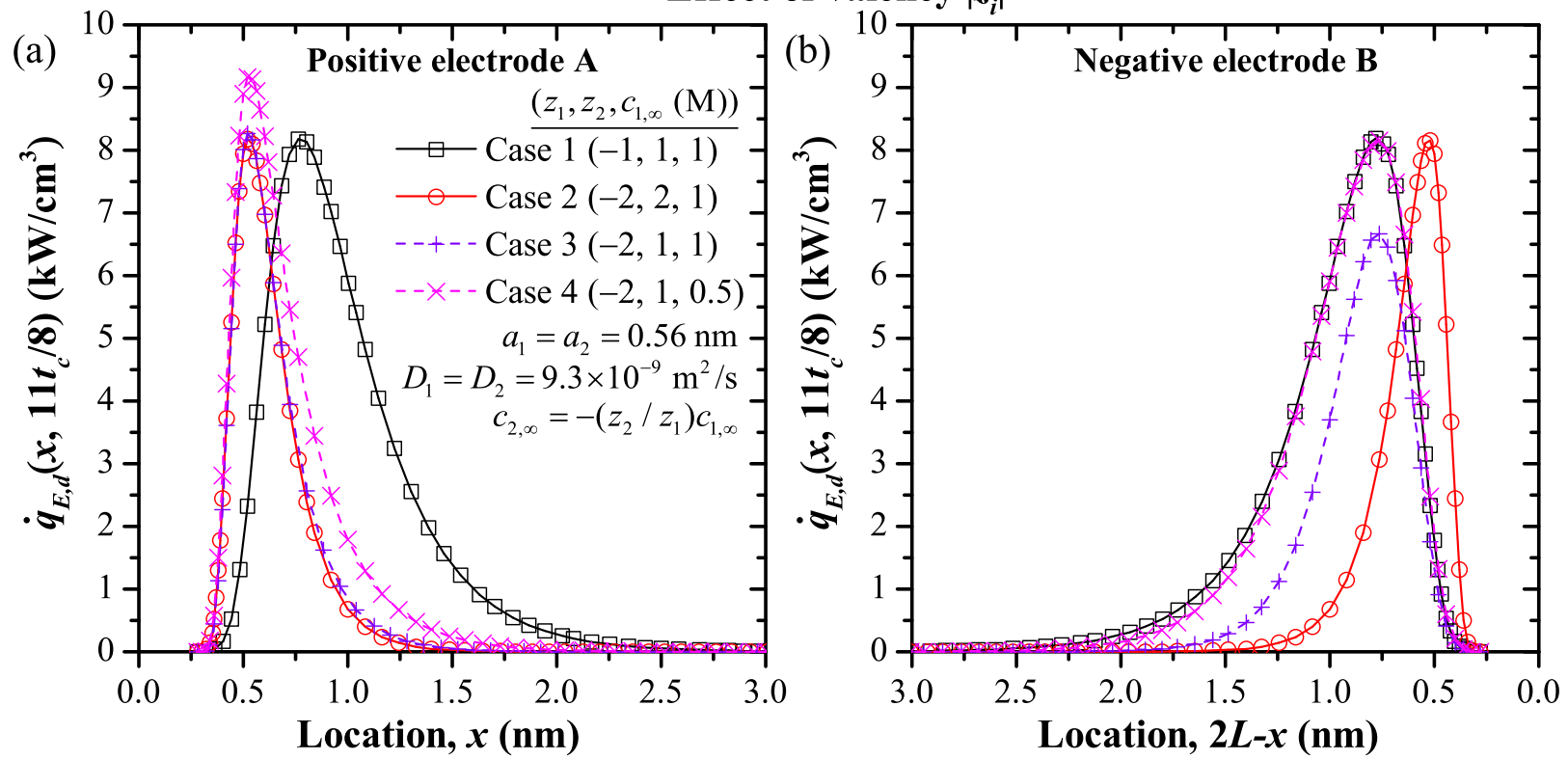

Effect of ion diameter $\boldsymbol{a}_{i}$
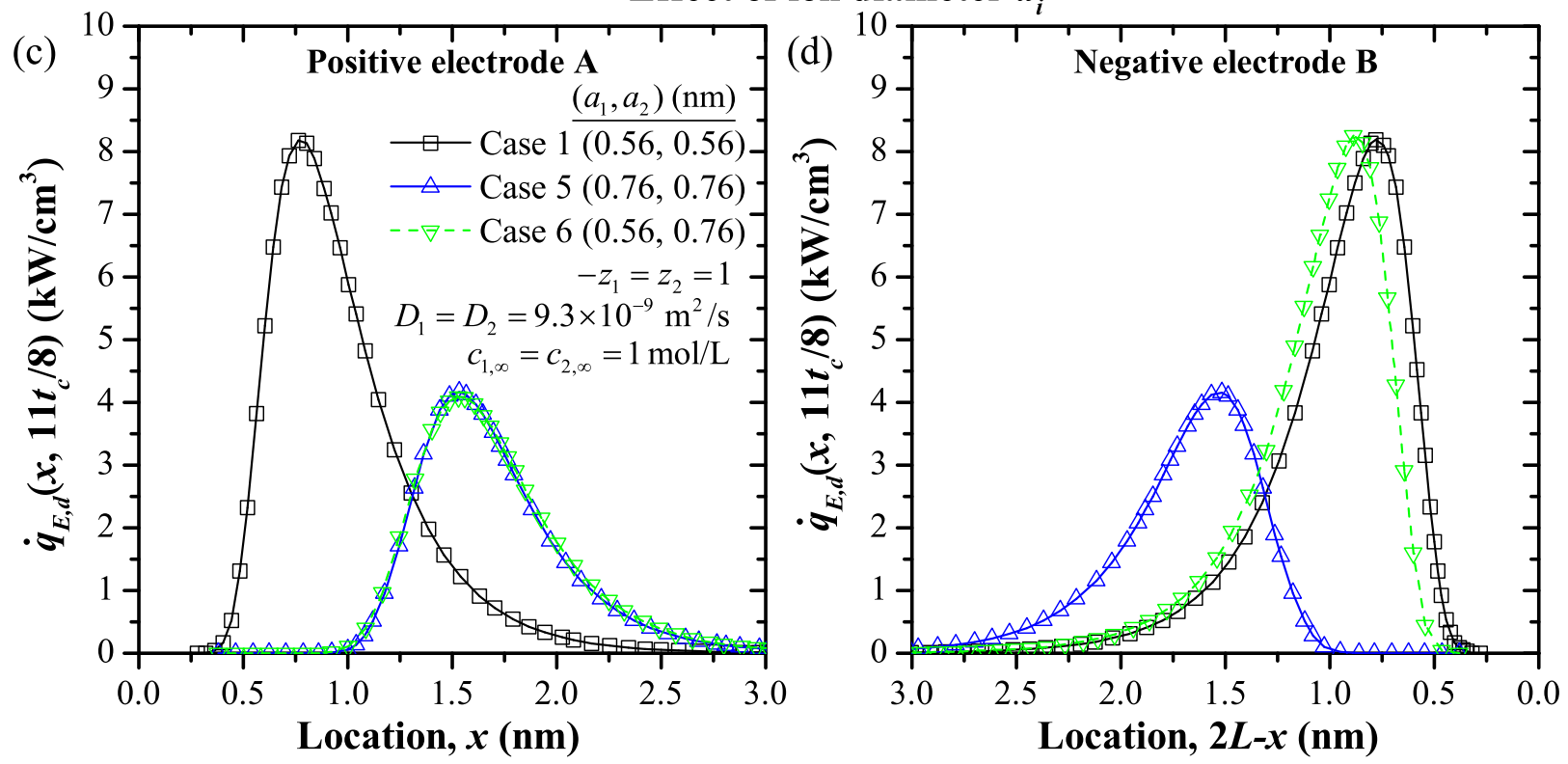

Figure 4: Diffusion heat generation rate $\dot{q}_{E, d}\left(x, 11 t_{c} / 8\right)$ as a function of location (a) and (c) $x$ near the positive electrode A and (b) and (d) $2 L-x$ near the negative electrode B. Plots (a) and (b) show Cases 1-4 with different $z_{i}$ while plots (c) and (d) show Cases 5 and 6 with different $a_{i}$. All cases correspond to time $t=11 t_{c} / 8$ near the end of a charging step. 
Effect of valency $\left|z_{i}\right|$
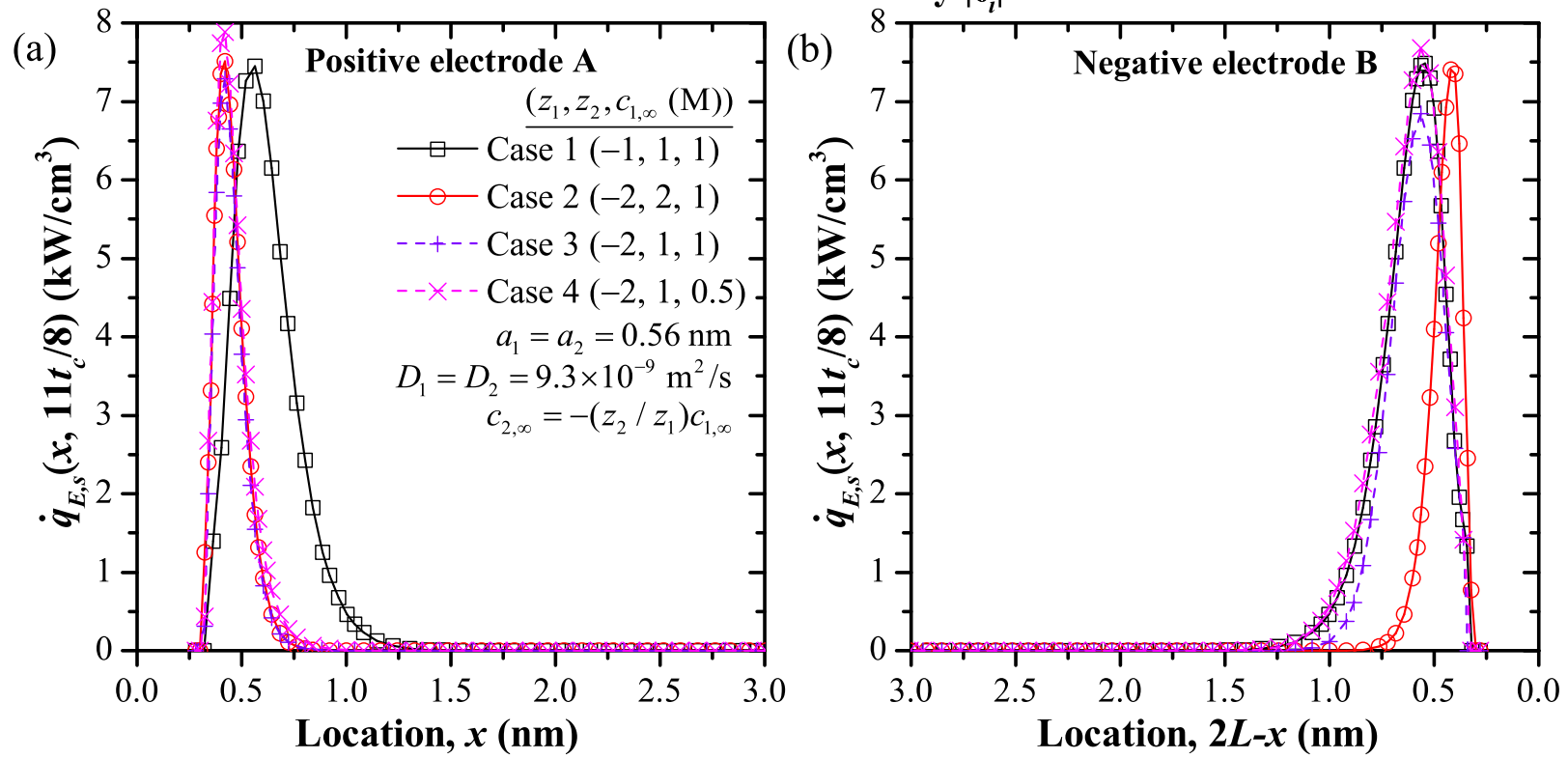

Effect of ion diameter $\boldsymbol{a}_{\boldsymbol{i}}$
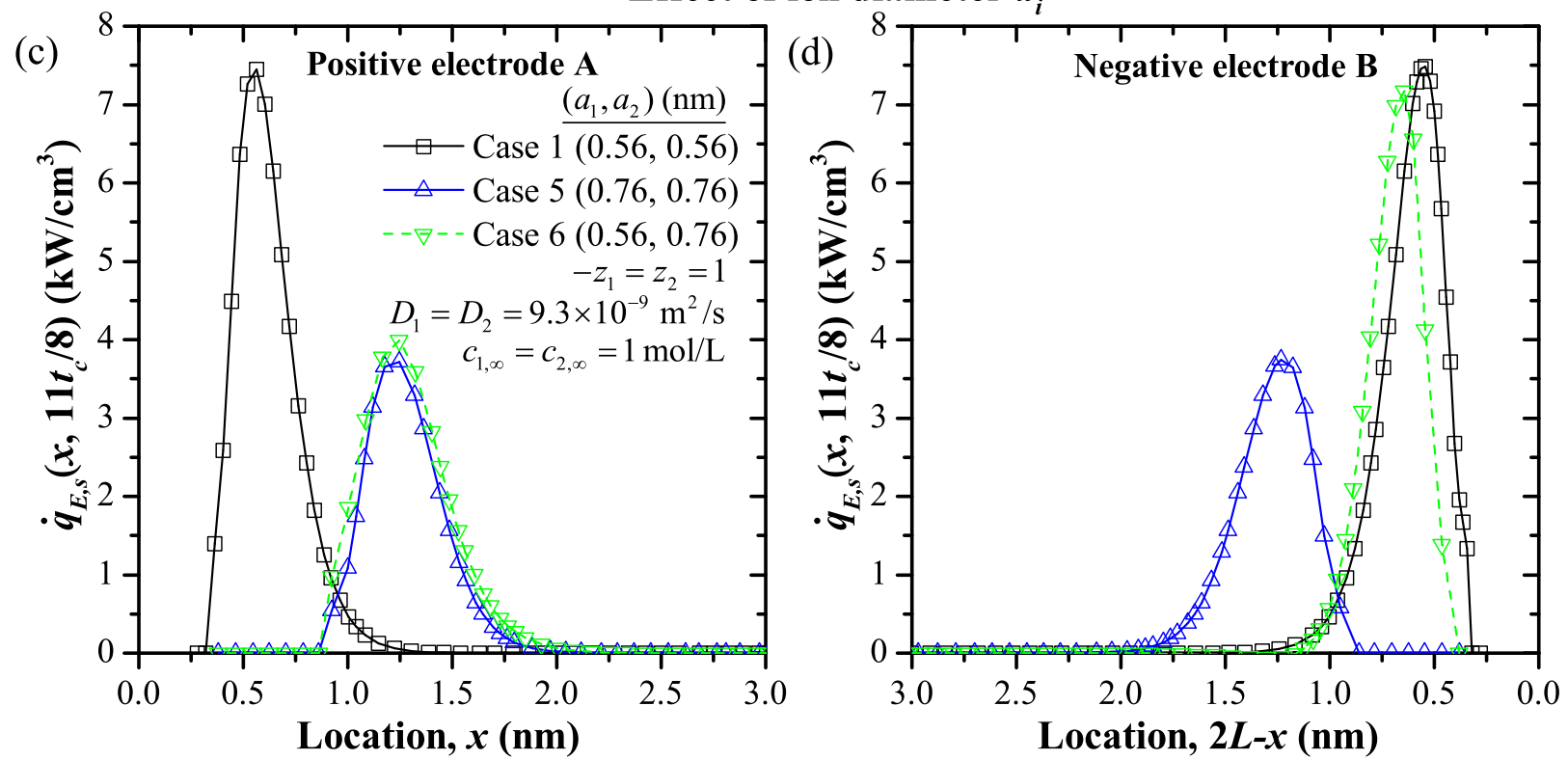

Figure 5: Steric heat generation rate $\dot{q}_{E, s}\left(x, 11 t_{c} / 8\right)$ as a function of location (a) and (c) $x$ near the positive electrode A and (b) and (d) $2 L-x$ near the negative electrode B. Plots (a) and (b) show Cases 1-4 with different $z_{i}$ while plots (c) and (d) show Cases 5 and 6 with different $a_{i}$. All cases correspond to time $t=11 t_{c} / 8$ near the end of a charging step. 
Effect of valency $\left|z_{i}\right|$
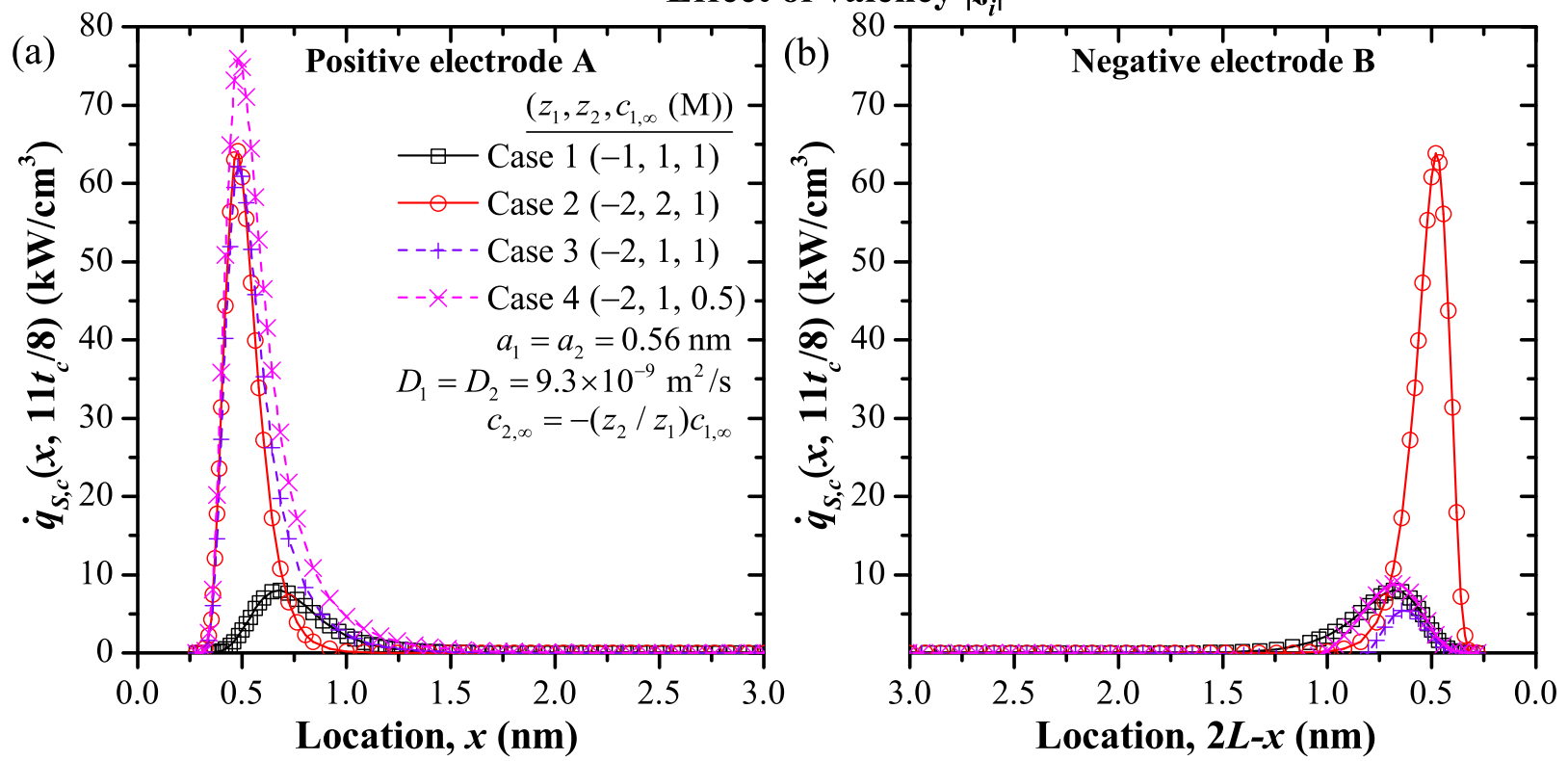

Effect of ion diameter $a_{i}$
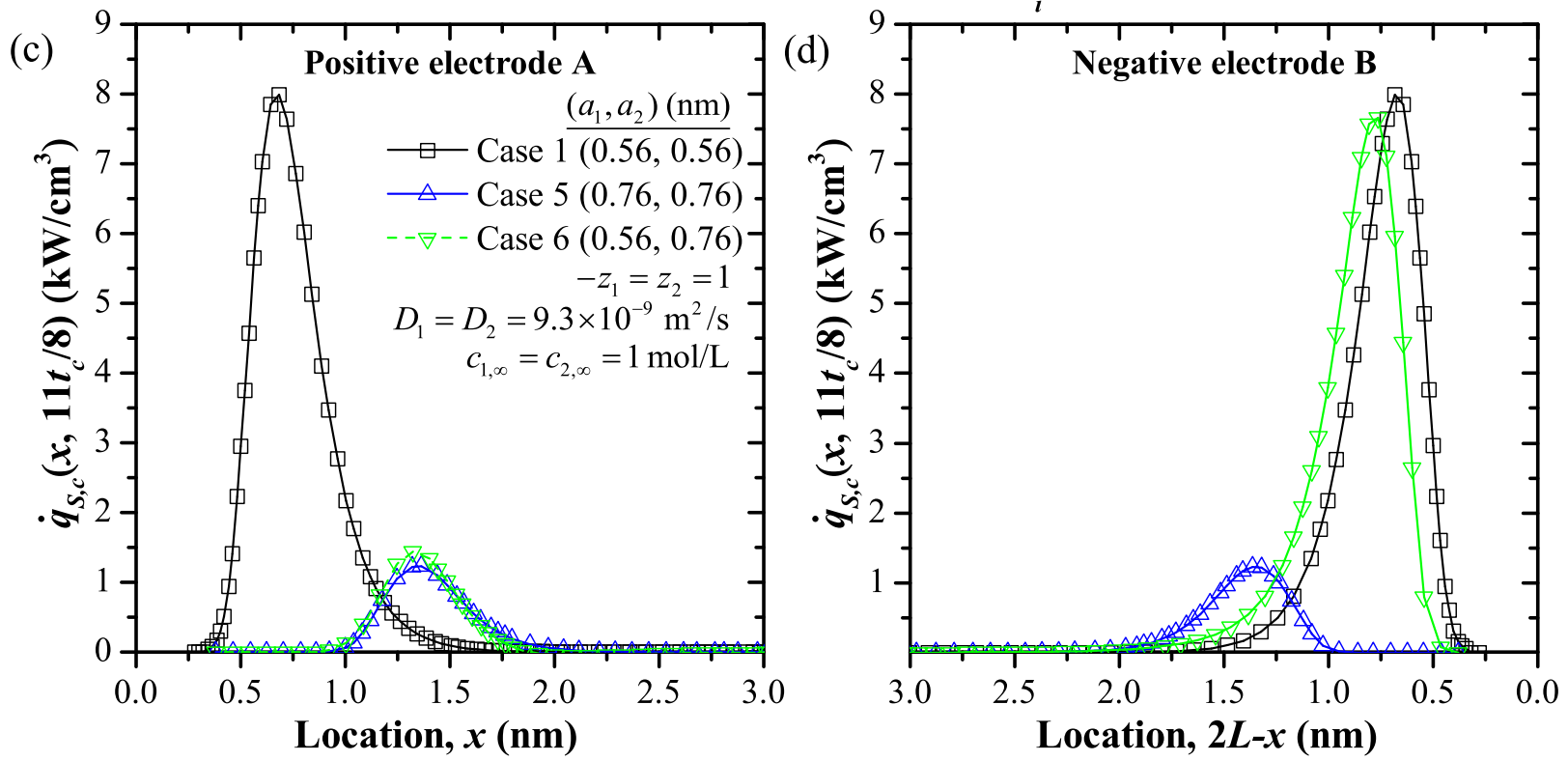

Figure 6: Heat of mixing heat generation rate $\dot{q}_{S, c}\left(x, 11 t_{c} / 8\right)$ as a function of location (a) and (c) $x$ near the positive electrode A and (b) and (d) $2 L-x$ near the negative electrode B. Plots (a) and (b) show Cases 1-4 with different $z_{i}$ while plots (c) and (d) show Cases 5 and 6 with different $a_{i}$. All cases correspond to time $t=11 t_{c} / 8$ near the end of a charging step. 
Effect of valency $\left|z_{i}\right|$
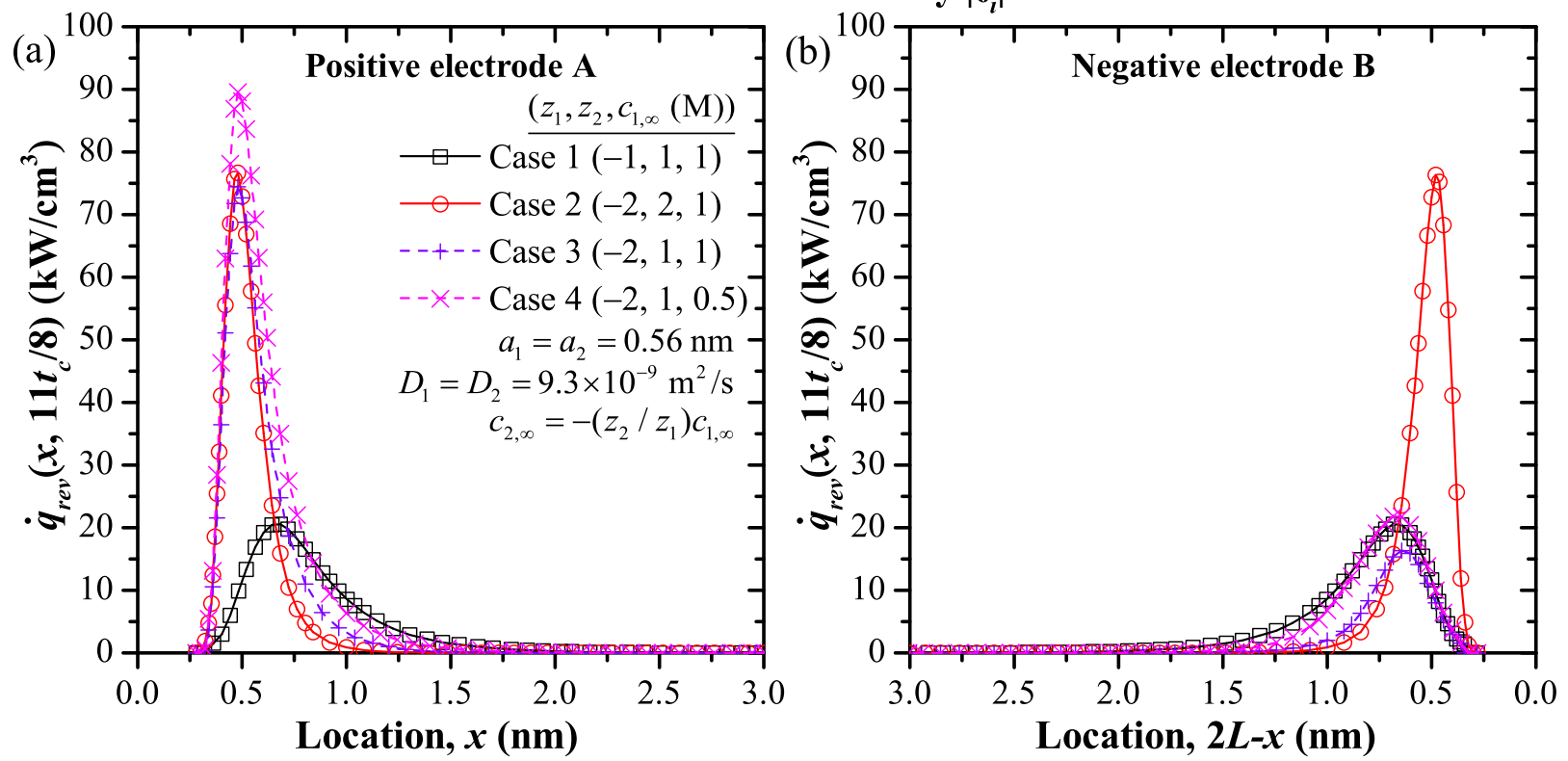

Effect of ion diameter $\boldsymbol{a}_{i}$
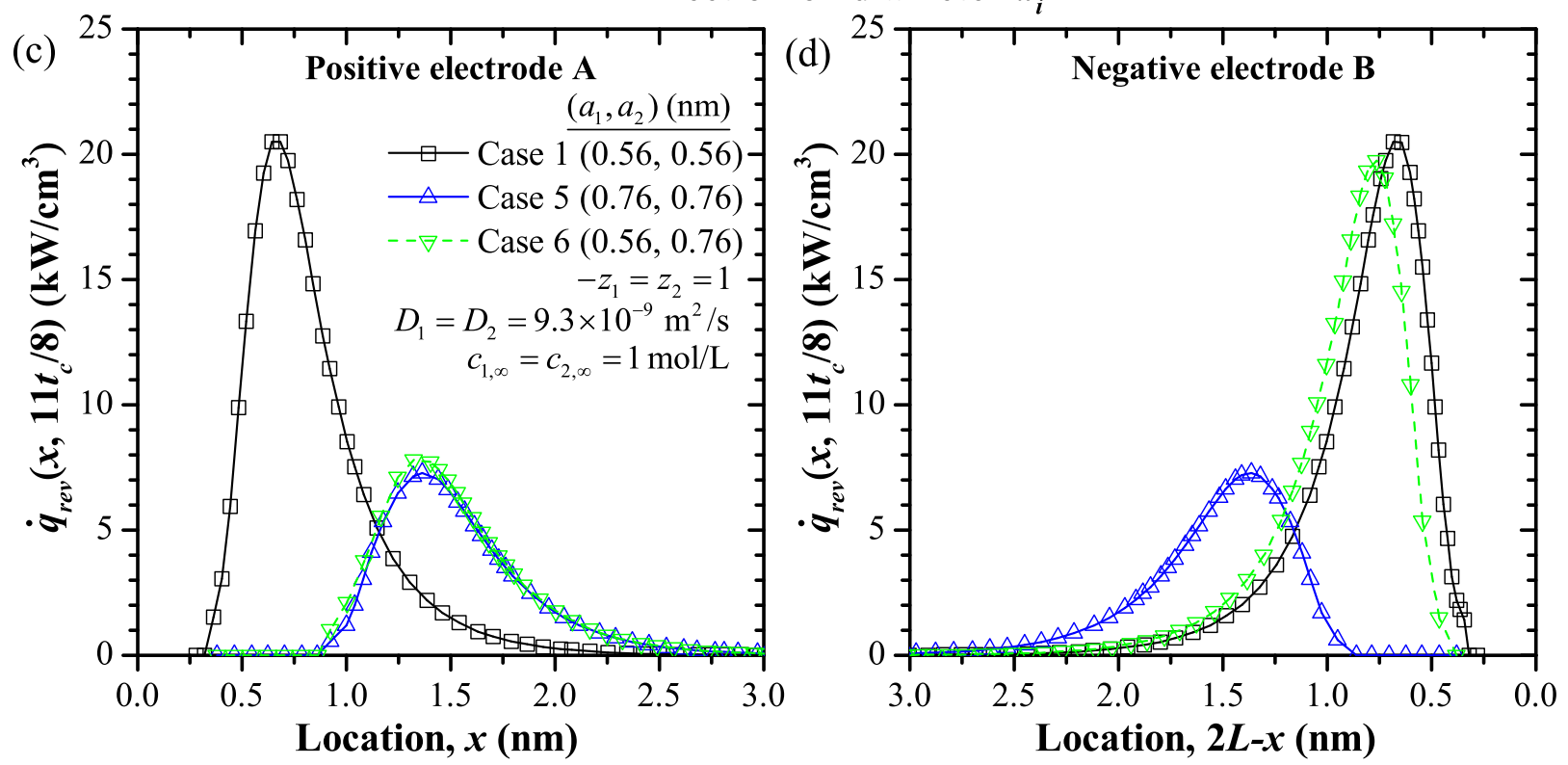

Figure 7: Overall reversible heat generation rate $\dot{q}_{r e v}\left(x, 11 t_{c} / 8\right)$ as a function of location (a) and (c) $x$ near the positive electrode A and (b) and (d) $2 L-x$ near the negative electrode B. Plots (a) and (b) show Cases 1-4 with different $z_{i}$ while plots (c) and (d) show Cases 5 and 6 with different $a_{i}$. All cases correspond to time $t=11 t_{c} / 8$ near the end of a charging step. 

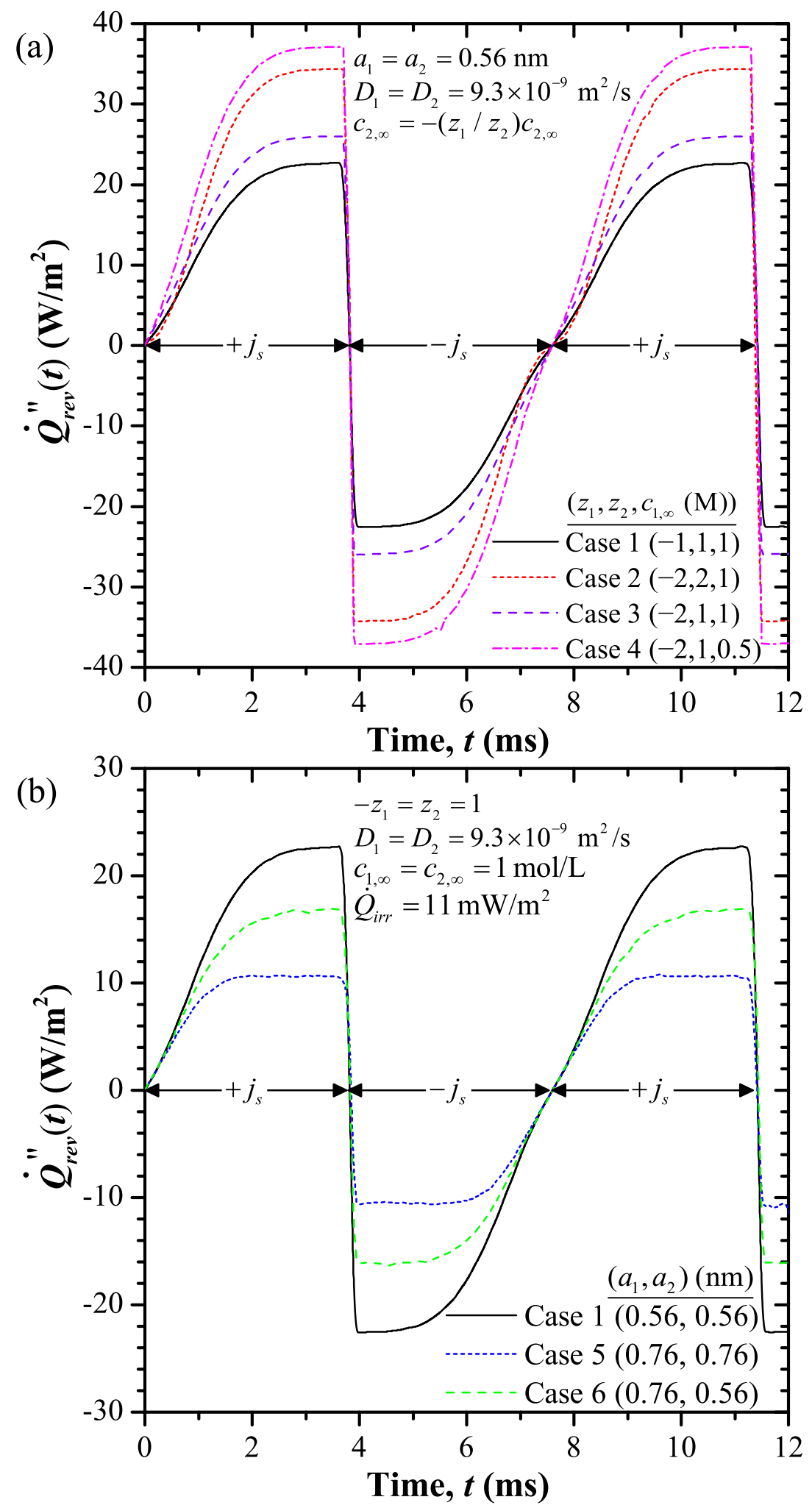

Figure 8: Total reversible heat generation rates $\dot{Q}_{r e v}^{\prime \prime}(t)$ as functions of $t$ over one and a half cycles for (a) Cases 1-4 with $a_{1}=a_{2}=0.56 \mathrm{~nm}, D_{1}=D_{2}=9.3 \times 10^{-9} \mathrm{~m}^{2} \mathrm{~s}^{-1}$, and different $z_{i}$ and (b) Cases 1, 5, and 6 featuring $-z_{1}=z_{2}=1, D_{1}=D_{2}=9.3 \times 10^{-9} \mathrm{~m}^{2} \mathrm{~s}^{-1}$, and different $a_{i}$. 
Positive electrode A
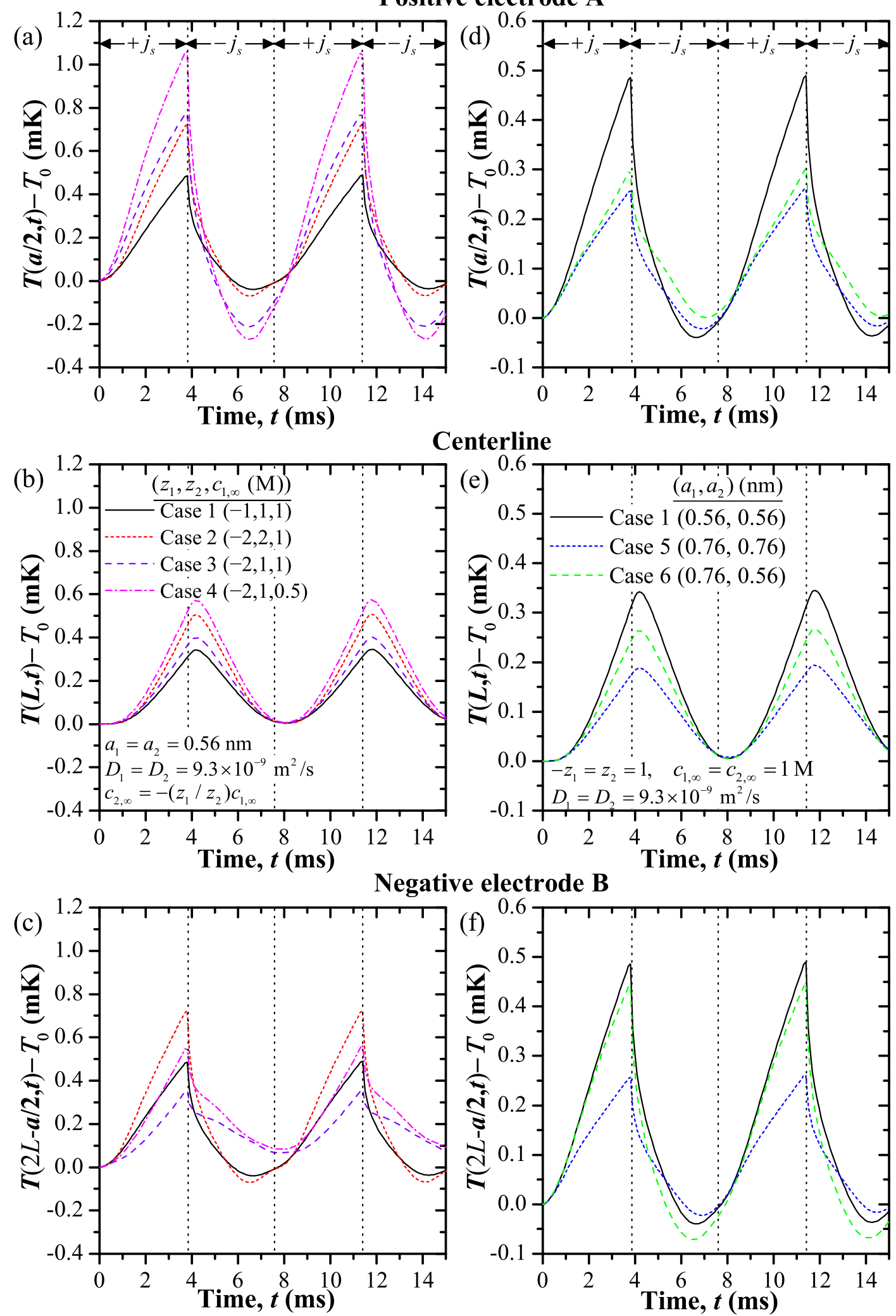

Figure 9: Temperature rise $T(x, t)-T_{0}$ as a function of time $t$ for (a)-(c) Cases 1-4 with different $z_{i}$ and (d)-(f) Cases 1, 5, and 6 with different $a_{i}$ for two consecutive cycles. Results are shown for three locations, namely, (a) and (d) at $x=a / 2$ near the positive electrode $\mathrm{A}$, (b) and (e) at the centerline $x=L$, and (c) and (f) at $x=2 L-a / 2$ near the negative electrode $\mathrm{B}_{38}$ 


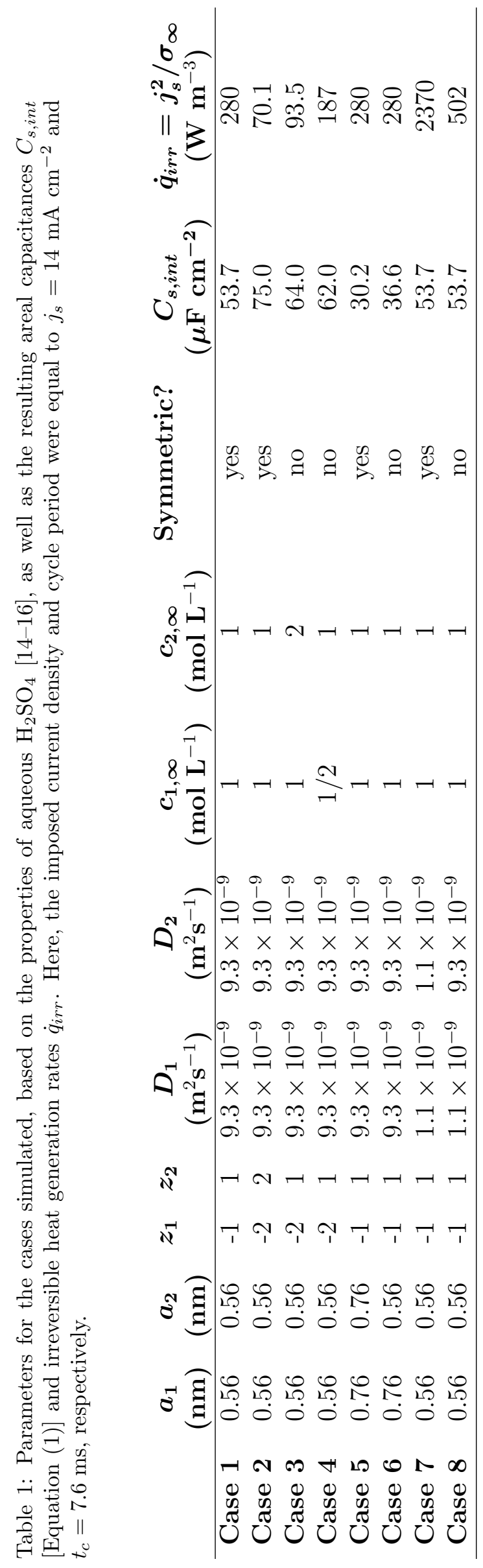


Table 2: Summary of the effects of changing ion properties on the heat generation rates $\dot{q}_{i r r}, \dot{q}_{r e v}, \dot{Q}_{i r r}^{\prime \prime}$, and $\dot{Q}_{r e v}^{\prime \prime}$ as well as the integral capacitance $C_{s, i n t}$ during galvanostatic cycling.

\begin{tabular}{|c|c|c|c|c|c|}
\hline & Increasing $\Rightarrow$ & $\begin{array}{c}\text { Diameter } \\
\quad a_{i}\end{array}$ & $\begin{array}{c}\text { Valency } \\
\left|z_{i}\right|\end{array}$ & $\begin{array}{c}\text { Diffusion coefficient } \\
\qquad D_{i}\end{array}$ & $\begin{array}{c}\text { Bulk concentration } \\
\boldsymbol{c}_{i, \infty}\end{array}$ \\
\hline$\dot{\boldsymbol{q}}_{\text {irr }}$ & & - & $\checkmark$ & $v$ & 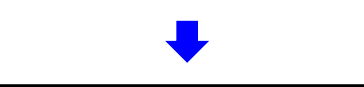 \\
\hline \multirow{2}{*}{$\dot{\boldsymbol{q}}_{\text {rev }}$} & counter-electrode & 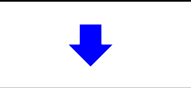 & 14 & - & 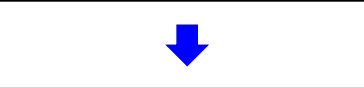 \\
\hline & co-electrode & - & — & - & 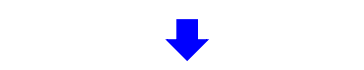 \\
\hline \multicolumn{2}{|c|}{$\dot{Q}_{i r r}^{\prime \prime}=2 L \dot{q}_{i r r}$} & - & $v$ & 7 & $v$ \\
\hline \multicolumn{2}{|l|}{$\dot{Q}_{r e v}^{\prime \prime}$} & 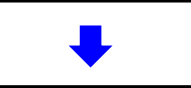 & 1 & - & 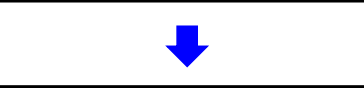 \\
\hline \multicolumn{2}{|c|}{$C_{s, i n t}$} & 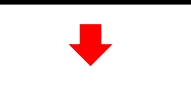 & 4 & - & 4 \\
\hline
\end{tabular}

